RICE UNIVERSITY

\title{
Operation of the Run IIB DØ Luminosity System and Determination of the Run IIB Luminosity Constant
}

\author{
by \\ Michelle V. Prewitt \\ A Thesis Submitted \\ in Partial Fulfillment of the \\ Requirements FOR The Degree \\ Master of Science
}

Approved, Thesis Committee:

Marjorie D. Corcoran, Chair

Professor of Physics and Astronomy

Karl Ecklund

Assistant Professor of Physics and

Astronomy

Thomas C. Killian

Associate Professor of Physics and

Astronomy

Houston, Texas

April, 2010 


\title{
Operation of the Run IIB DØ Luminosity System and Determination of the Run IIB Luminosity Constant
}

\author{
Michelle V. Prewitt
}

\begin{abstract}
The luminosity system is an integral part of the $\mathrm{D} \varnothing$ detector that must be properly maintained to provide accurate luminosity measurements for physics analysis. After the addition of a readout layer to the silicon vertex detector in 2006, it was necessary to re-calculate the effective inelastic cross section to which the luminosity monitor is sensitive. The preliminary analysis showed that the luminosity constant did not change with the addition of the extra layer of silicon. A full study of the revised luminosity constant including a complete analysis of systematic uncertainties has been completed. The luminosity constant was determined to be $\sigma_{\text {eff }}=48.3 \pm 1.9 \pm 0.6 \mathrm{mb}$, which reduces the $\mathrm{D} \varnothing$ contribution to the luminosity measurement uncertainty by almost $3 \%$.
\end{abstract}




\section{Acknowledgments}

I would like to thank . . . .

Marj Corcoran for all that she has taught me and for continuing to push me to work harder.

Yuji Enari for teaching me about the luminosity system, trusting me to work on it, and continuing to answer my questions when it misbehaves.

Rich Partridge for turning my months worth of tracking down detector material into something useful, helping with operations, and explaining the luminosity system over the phone in the middle of the night.

Mike Cooke for loads of latex help.

Mark for all his love and support.

My family and friends.

Thanks to all of you for being very patient. 


\section{Contents}

Abstract $\quad$ ii

Acknowledgments

List of Illustrations $\quad$ vi

List of Tables viii

1 Introduction 1

1.1 High Energy Particle Physics . . . . . . . . . . . . . . . . 1

1.2 Fermilab . . . . . . . . . . . . . . . . . . 4

1.3 DØ Detector. . . . . . . . . . . . . . . . 7

1.4 Physics and Luminosity _. . . . . . . . . . . . . . . . . . . . . 10

1.5 Inelastic Collisions . . . . . . . . . . . . . . . . . . . . . . . . 13

2 Luminosity System $\quad 15$

2.1 Luminosity Monitor . . . . . . . . . . . . . . . . . . . 15

2.1.1 Scintillator . . . . . . . . . . . . . . 17

2.1.2 Photo-Multiplier Tubes . . . . . . . . . . . . 18

2.2 Electronics Readout . . . . . . . . . . . . . . . . . . . . 19

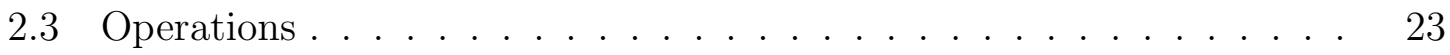


2.3.1 Calibrations ....................... 23

2.3.2 Monitoring ....................... 24

2.3.3 Maintenance.................. 26

\section{Run IIB Luminosity Constant 33}

3.1 Luminosity Measurement . . . . . . . . . . . . . . . . . 33

3.2 Luminosity Constant . . . . . . . . . . . . . . . . . . 35

3.3 Monte Carlo Simulation Samples . . . . . . . . . . . . . . 37

3.3.1 Run IIB Upgrades to the Monte Carlo Simulation . . . . . . . 37

3.3.2 Monte Carlo Simulation Sample Overlays . . . . . . . . . . . 43

3.4 Data Samples . . . . . . . . . . . . . . . 46

3.5 Determining the Non-Diffractive Fraction . . . . . . . . . . . . . 49

3.6 Results for $\sigma_{\text {eff }} \ldots \ldots \ldots \ldots \ldots \ldots$. . . . . . . . . . . . 53

3.7 Systematic Studies . . . . . . . . . . . . . . . . 55

3.7.1 Uncertainty Associated with Data . . . . . . . . . 56

3.7.2 Uncertainty Associated with Monte Carlo Simulation . . . . . 57

3.8 Conclusions . . . . . . . . . . . . . . . . . . 62

$\begin{array}{ll}\text { Bibliography } & 64\end{array}$ 


\section{Illustrations}

1.1 Quarks, Leptons, and Guage Bosons . . . . . . . . . . . . . 2

1.2 Fermilab Accelerator Complex . . . . . . . . . . . . 5

1.3 Luminosity Plot During Store . . . . . . . . . . . . . . . 8

1.4 Tevatron Beam Structure . . . . . . . . . . . . . . . . . . 9

1.5 DØ Detector . . . . . . . . . . . . . . . . . 11

1.6 Inelastic Collision Model . . . . . . . . . . . . . . . . . . . . . . . . . 14

2.1 Luminosity Monitor . . . . . . . . . . . . . . . . . . . . 16

2.2 Luminosity Monitor Rapidity Coverage . . . . . . . . . . . . . . . . . 17

2.3 Scintillator Annealing During Dry Air Purge . . . . . . . . . . . . . 20

2.4 Luminosity Electronics Readout System . . . . . . . . . . . . . . 20

2.5 Luminosity Monitor Pre-Amplifier Boards . . . . . . . . . . . . . . 21

2.6 Time and Charge Distributions from LMExamine . . . . . . . . . . 26

2.7 ADC versus Luminosity Plot . . . . . . . . . . . . . . . . . . 27

2.8 High Voltage Scans . . . . . . . . . . . . . . . . . . . 28

2.9 Threshold Scans . . . . . . . . . . . . . . . . . . . . . . . . . . 29 
2.10 Setting Luminosity Channel High Voltage . . . . . . . . . . . . . 31

2.11 Radiation Damage to Scintillator . . . . . . . . . . . . . . . 32

3.1 DØ Silicon Detector . . . . . . . . . . . . . . . . . 38

3.2 DØ Material Model . . . . . . . . . . . . . . . . . . . . . . . . 40

3.3 Forward Material Model . . . . . . . . . . . . . . . . . . . . 41

3.4 Luminosity Monitor Material Model . . . . . . . . . . . . . . . . . . . 42

3.5 Scintillator Light Yield by Position . . . . . . . . . . . . . . . . . . 44

3.6 Monte Carlo Simulation Timing and Charge Tune . . . . . . . . . . 45

3.7 Two Diminsional Multiplicity Distribution . . . . . . . . . . . . 48

3.8 Low Luminosity Multiplicity Distributions . . . . . . . . . . . . 51

3.9 Chi Squared Fit for Non-Diffractive Fraction . . . . . . . . . . . . 51

3.10 Preliminary Run IIB Non-Diffractive Fraction . . . . . . . . . . . . 52

3.11 Run IIB Non-Diffractive Fraction . . . . . . . . . . . . . . . . 54

3.12 Multiplicity Distribution for Nominal Geometry . . . . . . . . . . 59

3.13 Multiplicity Distribution for Tin Beam Pipe . . . . . . . . . . 60

3.14 Multiplicity Distribution for Pythia Parameter . . . . . . . . . 61 


\section{Tables}

3.1 Monte Carlo Simulation Acceptances . . . . . . . . . . . . . . . . 36

3.2 NTuple Data Samples . . . . . . . . . . . . . . . . . . . 47

3.3 Systematic Uncertainties . . . . . . . . . . . . . . . . . 63 


\section{Chapter 1}

\section{Introduction}

\subsection{High Energy Particle Physics}

High Energy Particle Physics studies the basic building blocks of the universe. The average person is aware that the material around them is composed of atoms. Atoms are composed of electrons that surround a nucleus because they are bound together by an electromagnetic force. The nucleus is a collection of protons and neutrons, which are made up of quarks that are bound together by the strong force. The goal of high energy particle physics research is to determine what particles are the basic building blocks of matter and how they interact with each other through the fundamental forces.

The current working theory that is strongly supported by experimental evidence is called the Standard Model [1]. This model explains the structure and interactions of particles through the basic building blocks of quarks, leptons, and the force-carriers, or gauge bosons. In the Standard Model, there are six quarks and six leptons which are shown in Figure 1.1. The quarks and leptons are grouped into three generations where successive generations mimic the properties of the previous generation but with

higher mass particles. The first generation particles, which are the most prevalent 


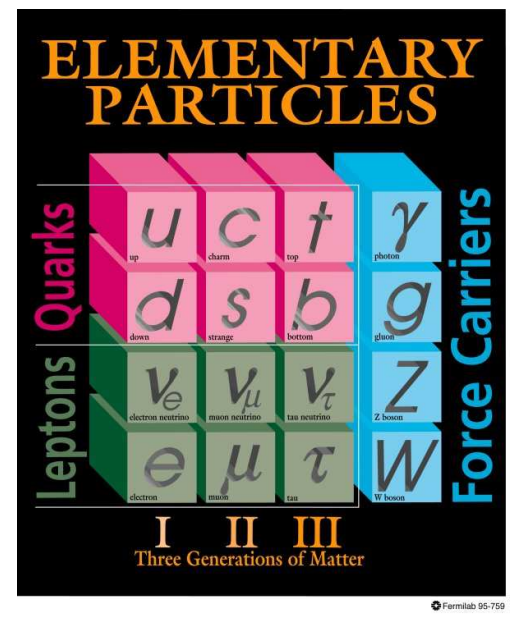

Figure 1.1 : The Standard Model states that matter is comprised of quarks and leptons, which interact through the guage bosons. (from Fermilab)

in nature, include the up $(u)$ and down $(d)$ quark and the electron $(e)$ and electron neutrino $\left(\nu_{e}\right)$. The first quark in each generation has a charge of $+2 / 3$ while the second quark in each generation has a charge equal to $-1 / 3$ in units of the electron charge. The first lepton in each generation has a charge of -1 while the second lepton, the neutrino, is neutral. Quarks have another property called color, which is like a charge associated with the strong force, that can come in three varieties: red $(R)$, green $(G)$, and blue $(B)$. Particles also have an anti-particle equivalent with the same mass but opposite charge. The anti-quarks $(\bar{q})$ have anti-colors cyan $(\bar{R})$, magneta $(\bar{G})$, and yellow $(\bar{B})$. The quarks and leptons all belong to a group of particles called fermions which have $1 / 2$ integer spin.

The force-carrying particles belong to a group called bosons, which have integer values of spin. The photon $(\gamma)$ is massless and mediates the electromagnetic force 
while the massive $W^{ \pm}$and $Z^{0}$ bosons mediate the weak force. Gluons come in eight varieties to interact with the three quark colors and mediate the strong force. The electromagnetic force is felt by all particles with charge while the strong force is only felt by the quarks and gluons. Quarks are found in color-neutral bound states known as hadrons. They can combine in groups of three quarks with each quark having a different color to form a baryon or in quark/anti-quark pairs having opposite color to make a meson.

One major success of the Standard Model was the unification of the electromagnetic and weak forces. It was successful in predicting the masses and properties of the $W^{ \pm}$and $Z^{0}$ bosons. The Standard Model has also accurately predicted particles such as the top quark, which was discovered at Fermilab in 1995 [2]. However, the Standard Model is incomplete. It has many arbitrary parameters, such as particle masses and coupling constants, and is inconsistent at high energies. The Standard Model does not account for the magnitude of the observed matter/anti-matter asymmetry in the universe. Also, the model predicts the existence of the Higgs boson, as a consequence of the mechanism to generate the $W^{ \pm}$and $Z^{0}$ mass, which has yet to be discovered. Thus the search to understand the universe at the most basic level continues.

High energy particle physics gets its name because studying the smallest particles requires a great deal of energy and extremely large detectors and accelerator complexes. Fermi National Accelerator Laboratory, often referred to as Fermilab, is a 
fully functional high energy particle physics research site. Another accelerator laboratory, CERN, houses the next generation research facility, the Large Hadron Collider (LHC). The LHC has initiated first collisions but is still in the tuning phase. For interactions to take place, particles must be loaded into the beam pipe, accelerated, and then collided at the detector halls. In these collisions, conditions mimic those soon after the beginning of the universe. By studying the particles created from the interaction of the two beams, particle physicists strive to determine fundamental laws and forces that govern the interactions of elementary particles.

\subsection{Fermilab}

Fermi National Accelerator Laboratory is located in Batavia, Illinois. Currently the Fermilab accelerator complex, seen in Figure 1.2, boasts the highest intensity hadron accelerator and the highest energy proton-antiproton collider in the world. The accelerator at Fermilab that makes high energy particle physics research possible is the Tevatron, an accelerator consisting of hundreds of superconducting magnets located in a $1 \mathrm{~km}$ radius ring. However, it takes many steps before collisions can occur in the Tevatron. One way the Tevatron is unique is that it collides protons $(p)$ and antiprotons $(\bar{p})$. Protons are prevalent in nature and can be easily collected. Anti-protons are the anti-matter equivalent to protons, which annihilate with matter. Thus antiprotons have to be produced and carefully stored at Fermilab. To make the proton beam, hydrogen ions with two electrons are accelerated through a Crockoft-Walton 


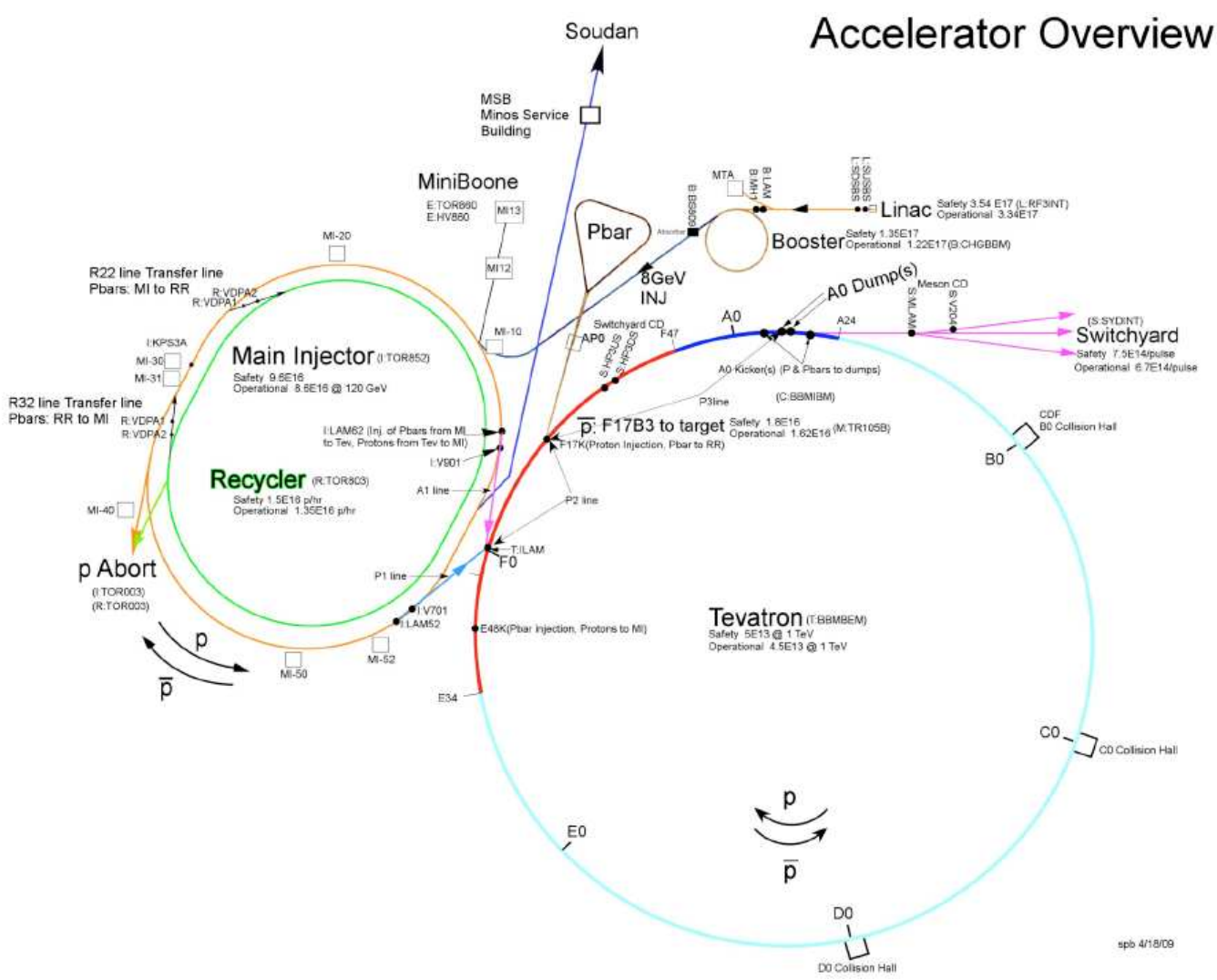

Figure 1.2 : Schematic of the Fermilab Accelerator Complex. The Tevatron is the highest energy $p \bar{p}$ collider in the world. The $1 \mathrm{~km}$ radius ring houses hundreds of superconducting magnets to acclerate the beams to a center of mass energy of $\sqrt{s}=1.96 \mathrm{TeV}[3]$. 
device and then a linear accelerator before the electrons are stripped off. The protons are then moved to the Booster where they are accelerated from an energy of $400 \mathrm{MeV}$ to $8 \mathrm{GeV}$. After the Booster a $p$ travels to the Main Injector where it waits to be put into the Tevatron. Collecting anti-protons takes much more effort. To make antiprotons, $120 \mathrm{GeV}$ protons from the Main Injector are shot into a nickel target. In this collision, anti-protons are created as a secondary particle which are collected at a rate of $2 \bar{p}$ per $10^{5} p$ on target [4]. The anti-protons are collected by the Debuncher and stored in the Accumulator where the particles are also stochastically cooled. In order to maximize the efficiency of $\bar{p}$ production, anti-protons are stored in the Accumulator until a group can be transferred to the Recycler. During normal operations, a transfer of about 25E9 anti-protons is made every hour. The anti-protons are stored in the Recycler at an energy of $8 \mathrm{GeV}$, where they are further cooled through electron cooling, until the Tevatron is ready for a new set of particles. Then the anti-protons are transferred to the Main Injector where the energy is increased to $150 \mathrm{GeV}$, and the particles can be moved into the Tevatron [3].

The period of time during which a single load of protons and anti-protons is in the Tevatron is called a store, an example of which is shown in Figure 1.3. A typical store length is from 12 to 20 hours. Protons and anti-protons are injected into the Tevatron from the Main Injector in groups rather than a continuous stream. The Tevatron has 159 ticks, or windows, where particles can be placed. Of these only 36 ticks have beam injected into them. A tick with beam is referred to as a bunch. Each 
bunch is separated by at least 2 empty ticks. Groups of 12 bunches, or super-bunches, are separated by a larger numbers of empty ticks as shown in Figure 1.4. The 3 large gaps are referred to as abort gaps, since they allow time for the beam to be safely aborted in case of problems. An orbit around the tunnels for a bunch is called a turn. The beam orbits the Tevatron at a rate of about $47 \mathrm{kHz}[5]$.

Collisions occur at only select points around the Tevatron. Two experiments sit at interaction points on the Tevatron to study the physics that results from the high energy collisions. The Collider Detector at Fermilab (CDF), was built early in the history of the Tevatron. D-Zero (DØ), which is named for the interaction point where it is located, was constructed a bit later. Each experiment uses a multi-purpose particle detector to gain as much information as possible about the $p \bar{p}$ collisions.

\subsection{DØ Detector}

The $\mathrm{D} \varnothing$ detector is actually a combination of many sub-detectors that work together to allow each interaction between a colliding $p$ and $\bar{p}$ to be studied. The sub-detectors gather information from the secondary particles that are created in beam collisions. The sub-detectors are layered within each other around the interaction point, which is in the beam pipe approximately in the middle of the detector. Figure 1.5 is a schematic of the $\mathrm{D} \varnothing$ detector. When moving away from the beam pipe, a particle would first hit the Silicon Microstrip Tracker (SMT). The SMT is comprised of solid-state silicon pieces that are very useful in following tracks created by particles 


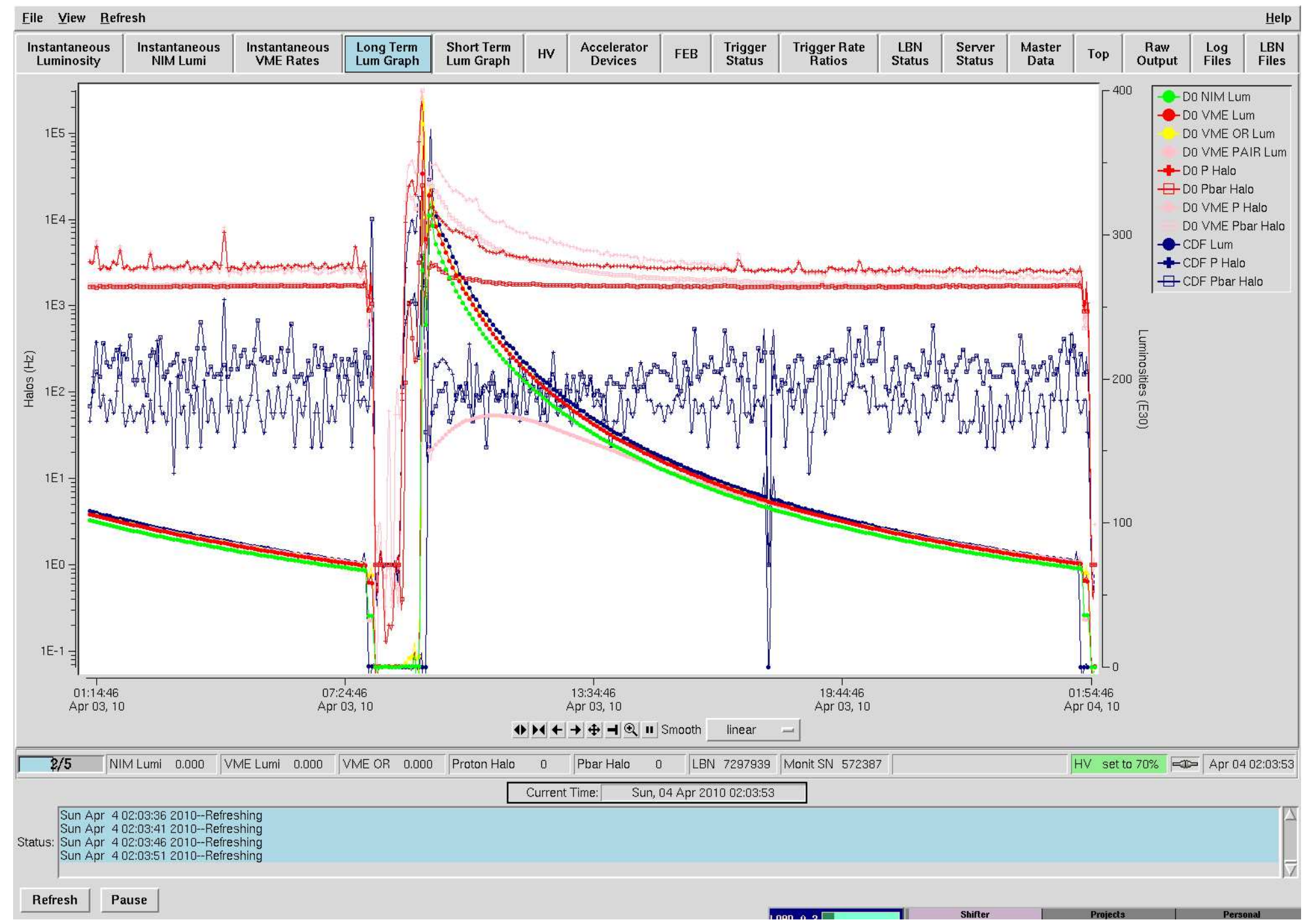

Figure 1.3 : GUI used by luminosity experts to track the luminosity and halo values during store. The plot is for store 7725 which lasted approximately 16 hours and went from a luminosity of 335E30 to 75E30. 


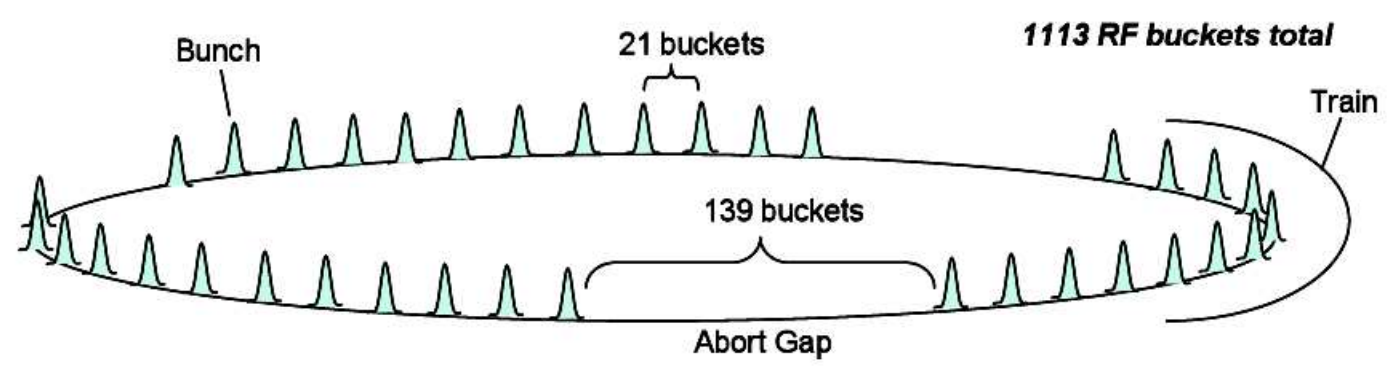

Figure 1.4 : The 36 bunches in the Tevatron are grouped into sets of 3 super-bunches seperated by abort gaps [5].

and determining particle vertices, the place where a particle decays into more stable particles. Moving away from the interaction region, the next sub-detector is the Central Fiber Tracker (CFT). The CFT tracks longer-lived particles after they pass through the SMT. The CFT and SMT sit inside the magnetic field of a solenoid. The tracks produced in the CFT are useful for determining particle momentum because of the curvature of charged particles in a magnetic field. The Central Preshower (CPS) lies between the CFT and Calorimeter, outside the solenoid. The purpose of the preshower is to initiate electromagnetic showers to help identify electrons and photons. The Calorimeter is the next sub-detector moving away from the interaction point at $\mathrm{D} \varnothing$. The Calorimeter is divided into three sections: electromagnetic, fine hadronic, and coarse hadronic. Each section is comprised of liquid argon and different metals making the Calorimeter useful in determining the type and energy of particles. The final full coverage sub-detector at DØ is the Muon System. The main purpose of this detector is to identify muons after they escape the inner sub-detectors in DØ. DØ also has a special sub-detector called the luminosity monitor. It sits in a very 
forward region and is used for determining the luminosity of the beam [6].

A vast amount of information is produced by reading out the sub-detectors during physics data taking. It is important to have a good triggering system so that only interesting events are kept for later analysis. The first decisions as to what events to keep are made at the hardware level for each sub-system. After the Level 1 (L1) cut is made, information from sub-detectors starts to be grouped and a Level 2 trigger (L2) is applied. L1 reduces the information to a rate around $2 \mathrm{kHz}$, and $\mathrm{L} 2$ cuts that rate in about half. Then the data is sent to the Level 3 (L3) farm where the information rate is reduced to $100 \mathrm{~Hz}$ to be written to tape. In contrast, the luminosity data acquisition system (DAQ) records information from all bunch crossings, and no cuts are made to reduce the amount of data [6].

\subsection{Physics and Luminosity}

$\mathrm{CDF}$ and $\mathrm{D} \varnothing$ are multi-purpose particle physics detectors designed to study many different types of particles and glean a variety of information about each particle detected. The different components of each detector are used to identify information including particle type, charge, energy and momentum. From this information, interactions are traced back and re-built to determine particle decay chains, lifetimes, and masses. True understanding is gained by comparing experimental evidence with theoretical predictions. Finding a new particle, predicted or not, is one way to validate or disprove a theory. Another way to test a theory is by comparing experimental and 


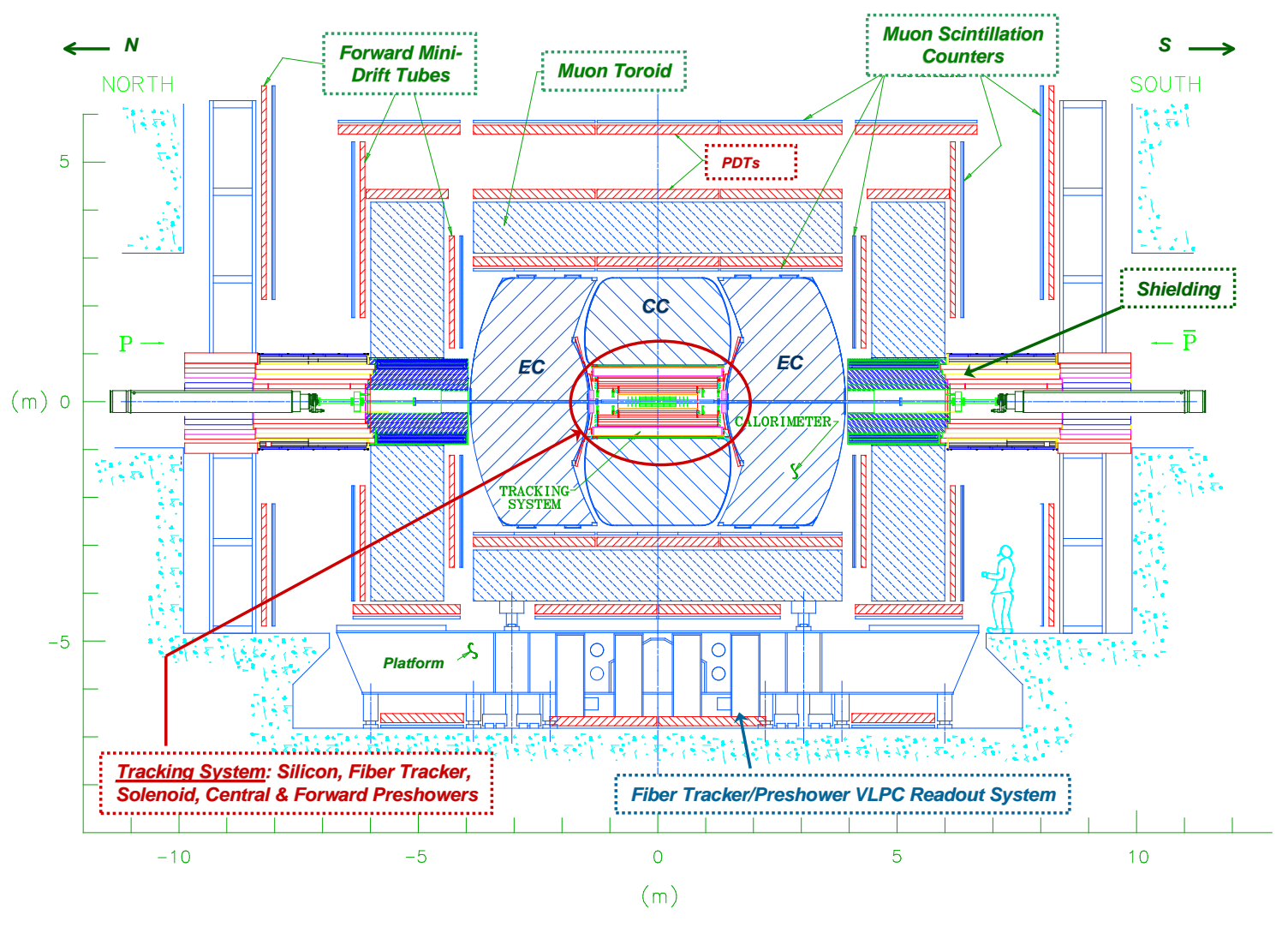

Figure 1.5 : Schematic of the DØ Detector. The detector combines information from the different sub-detectors to collect data for multi-purpose physics analysis. (from A. Heinson) 
predicted cross section measurements.

A cross section is calculated by determining the rate at which the event occurs and normalizing this rate by the luminosity of the data sample. The general relationship between event rate $(\mathrm{N})$ in interactions per second, cross section $(\sigma)$, and luminosity $(\mathrm{L})$, is given in Equation 1.1.

$$
N=\sigma L
$$

A cross section has units of area or $\mathrm{cm}^{2}$, and the luminosity has units of $\mathrm{cm}^{-2} \mathrm{~s}^{-1}$. At Fermilab, the luminosity is a measure of how often protons and anti-protons collide at the interaction points. Luminosity depends on the number of protons and antiprotons as well as how tightly the bunches are packed. The DØ luminosity monitor is a unique part of the detector, the sole purpose of which is to measure the luminosity for each bunch crossing. This sub-detector determines characteristics of the beams that are colliding, rather than collecting information about the particles produced by the collisions. All the other sub-detectors at $\mathrm{D} \varnothing$ collect information about the events produced by the $p \bar{p}$ interaction. Thus all the other sub-detectors work together so that an event rate can be measured. With the event rates determined by the other sub-detectors and the luminosity calculated from the luminosity monitor, researchers at $\mathrm{D} \varnothing$ are able to make precision cross section measurements. 


\subsection{Inelastic Collisions}

The luminosity monitor sits in a forward region of the DØ detector where it is sensitive to hits by secondary particles created through inelastic collisions during beam crossings. The model used to describe the inelastic events breaks interactions down into three subsets: Non-Diffractive (ND), Single Diffractive (SD), and Double Diffractive (DD). In each type of event, low transverse momentum particles are produced. Particles traveling in the forward direction at small angles with respect to the beam direction have the potential to be detected by the luminosity monitor. For single diffractive events, only one of the original particles in the interaction breaks up. In both non-diffractive and double diffractive events both particles break up and have the potential to send multiple particles in the forward direction. The difference between a double diffractive and non-diffractive event is that a double diffractive event produces very few particles at angles near 90 degrees from the beam. However, both ND and DD types of events appear similar in the luminosity monitor. Figure 1.6 shows examples of the different types of inelastic events. The effects of the different types of inelastic events on the luminosity measurement will be discussed in more detail in Chapter 3. 


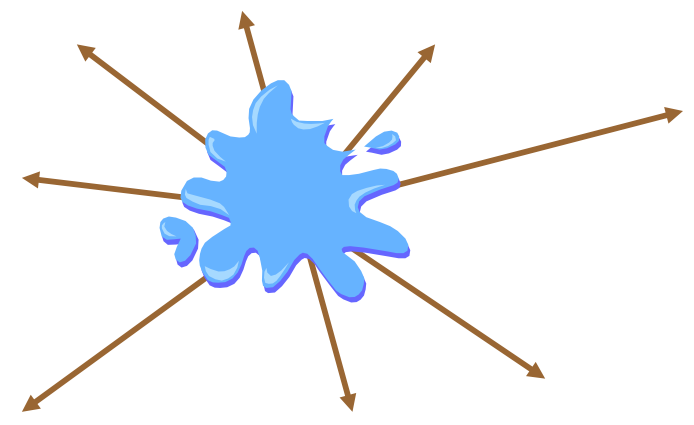

(a) Non-Diffractive

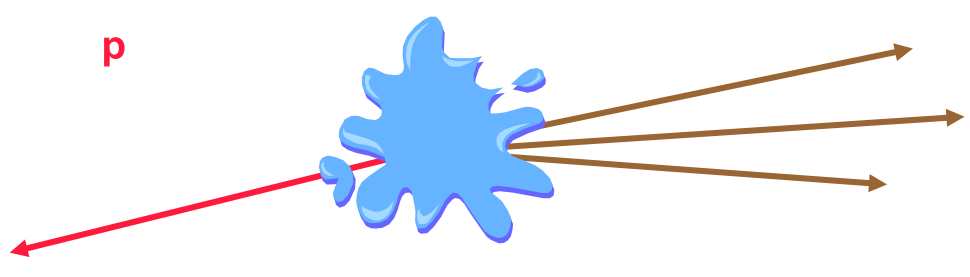

(b) Single Diffractive

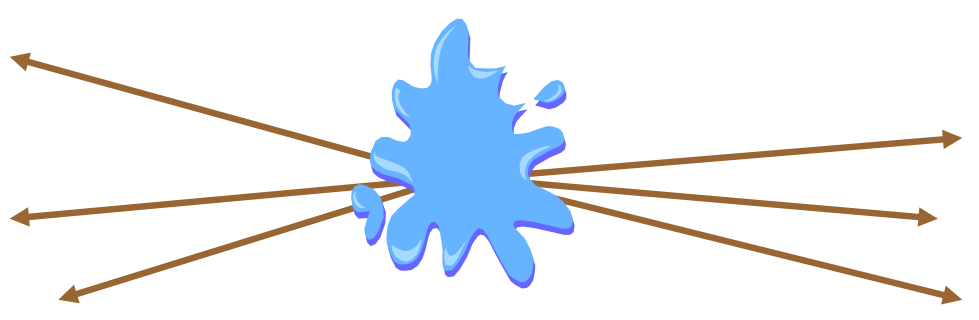

(c) Double Diffractive

Figure 1.6 : Cartoons of the three types of inelastic events for the model used in the analysis. The splash represents the $p \bar{p}$ interactions and the arrows show the trajectories of particles created from the collision. Forward or backward moving particles have the potential to hit the luminosity monitor. (from R. Partridge) 


\section{Chapter 2}

\section{Luminosity System}

\subsection{Luminosity Monitor}

The DØ luminosity monitor consists of two scintillator arrays each with 24 channels. A channel consists of a scintillating wedge with a photo-multiplier tube (PMT) attached. The two arrays sit in the forward region of the $\mathrm{D} \varnothing$ detector at $\pm 140 \mathrm{~cm}$ from the center of the detector where the beams collide. Each array surrounds the beam pipe in a donut shape with the scintillator wedges perpendicular to the direction of particles traveling along the beam pipe. The two arrays are distinguished as North, the side through which the protons enter into the detector, and South, where the anti-proton pass first. Figure 2.1 shows the North enclosure surrounding the beam pipe. This geometry allows the luminosity monitor to cover a pseudo-rapidity range of $2.7<|\eta|<4.4$, as seen in Figure 2.2. Pseudo-rapidity, written as $\eta$ and shown in Equation 2.1, is a measure of the angle of trajectory of a particle with the beam direction as the $\theta=0$ reference [7].

$$
\eta=-\ln (\tan (\theta / 2))
$$




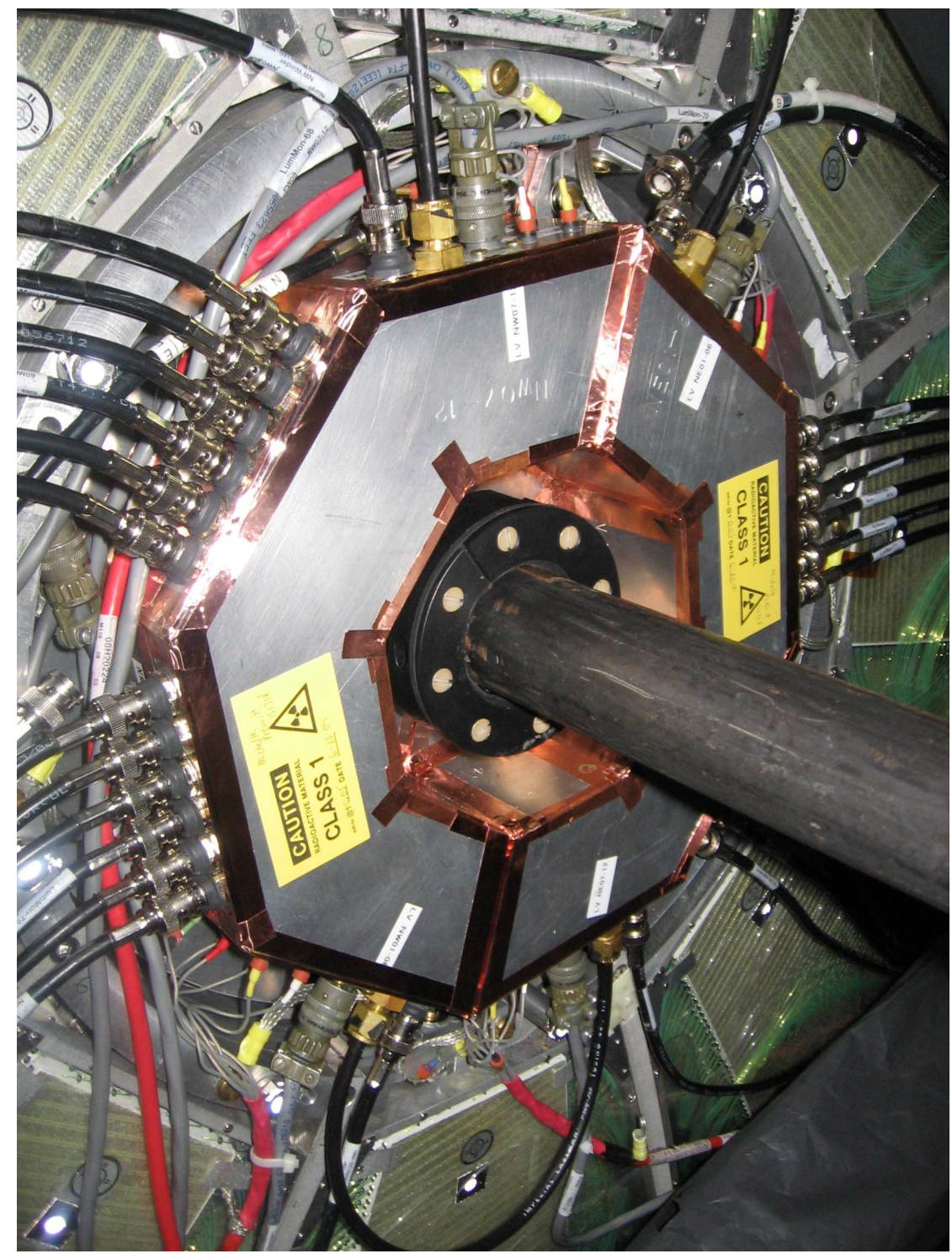

Figure 2.1 : Picture of the North luminosity monitor array taken in July 2009 at the end of a maintenance period. The gap between the End Cap and Central Calorimeter is closed during normal operations and this view is not possible. The scintillator, PMTs, and pre-amplifiers are all housed inside two aluminium casing, referred to as Northwest and Northeast, to allow the enclosures to be removed for maintenance. 


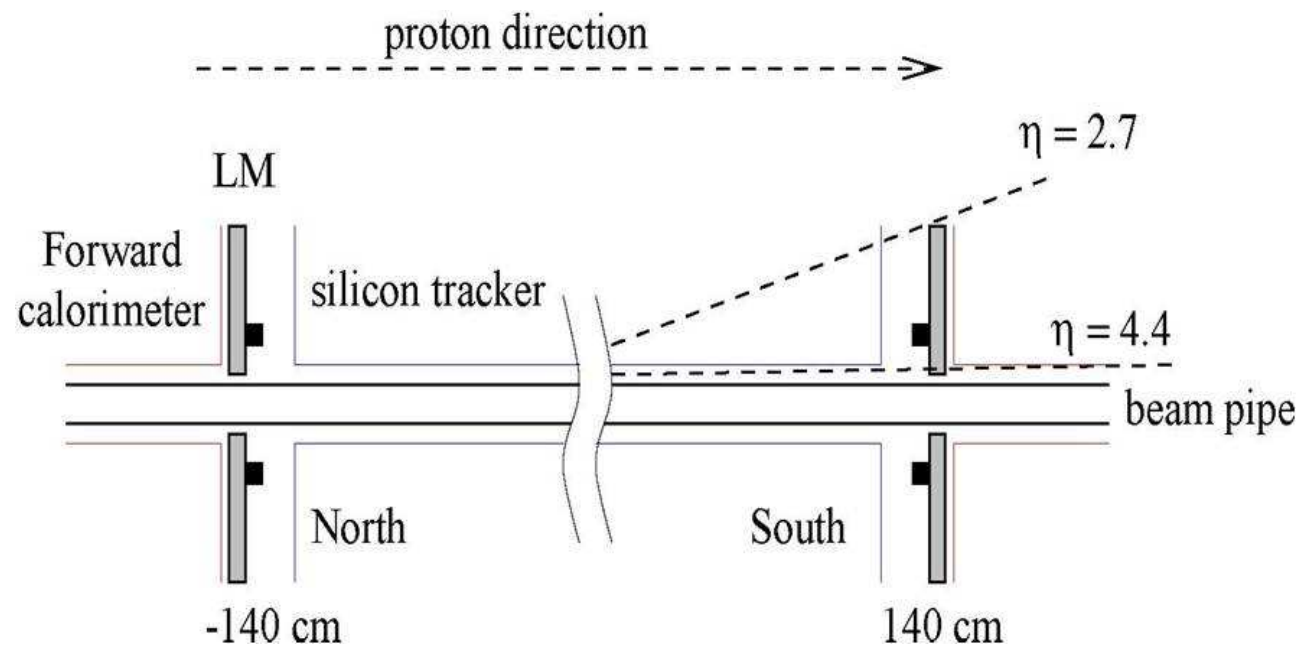

Figure 2.2 : A schematic showing the North and South luminosity monitor (LM) enclosures with respect to beam direction, position, and pseudo-rapidity coverage [7].

\subsubsection{Scintillator}

The scintillating wedges used in the luminosity monitor are Saint-Gobain BC-408. The wedges are cut to trapezoids with a length of 6 inches and parallel sides of 0.332 and 1.912 inches. The plastic is polyvinyltoluene, PVT, primarily doped with anthracene. As charged particles pass through the plastic, they lose energy through ionization. The PVT absorbs the energy, becomes excited, and emits photons in the UV spectrum. Anthracene is needed to shift the wavelength of these photons from UV to a visible blue light. The peak output wavelength of BC-408 is $425 \mathrm{~nm}$. The blue light travels through the scintillator wedge and is detected by the attached PMT [8]. 


\subsubsection{Photo-Multiplier Tubes}

The luminosity system uses Hamamatsu R7494 photo-multiplier tubes. These PMTs are 1" diameter, fine-mesh tubes with quartz windows, which are designed to run at negative high voltages. The tubes have a bialkali photocathode. The maximum sensitivity of the PMT occurs for light of wavelengths of approximately 400 to $500 \mathrm{~nm}$ $[9]$.

Custom PMTs are needed in the detector because of the conditions to which the PMTs are exposed. The placement of the luminosity monitor exposes the PMTs to the magnetic fringe field of the solenoid that is part of the $D \varnothing$ tracking system. The solenoid produces a 2 Tesla magnetic field at its center and has a field strength of approximately 1.25 Tesla at the location of the luminosity monitor [6]. PMTs with an ordinary dynode structure would not function properly in the luminosity monitor because the magnetic field would not allow the electrons to pass through the dynode structure, but electrons can pass through the mesh dynode structure of the customized PMTs. The PMTs were designed with a quartz window because it is more resistant than glass to damage from the radiation that bombards the detector. The disadvantage to the quartz window is that it is more permeable to helium. Since helium is used in abundance for cooling in the Tevatron and at D $\varnothing$, the luminosity monitor is constantly purged with dry gas, which keeps helium from settling in the luminosity enclosures, thus reducing the risk of damage to the tubes by helium infiltration. After testing in the Spring of 2007, the purge gas was switched from nitrogen 
to dry air since evidence from gain studies, see Figure 2.3, showed that the dry air purge anneals some of the radiation damage in the scintillator.

\subsection{Electronics Readout}

The scintillating wedge and PMT combination give a fast and accurate reading of particles passing through the luminosity monitor. The signal from each channel must be collected and processed to produce an accurate luminosity measurement that can be associated with events in the data. Figure 2.4 shows a schematic of the luminosity system readout.

The signal for each PMT is pre-amplified by a factor of 60.5 by electronics inside the luminosity monitor enclosures, shown in Figure 2.5. Then the analog signal moves via cables to racks outside the collision hall shielding. The readout crates for the luminosity system are housed inside the first floor of a moving counting house (MCH1). The signal from the luminosity monitor is split between the two types of electronics modules, NIM and VME, which are used by the luminosity system. While the NIM system was the standard for operation from 2000 to 2005, the VME system is now used to make the official luminosity measurement [10].

The NIM system is currently used for monitoring purposes and to calculate halo rates. Halo is created when particles from the bunches escape and interact with material around the beam pipe. If beam particles interact with surrounding material upstream of the $\mathrm{D} \varnothing$ detector, they have the potential to cause showers of particles 


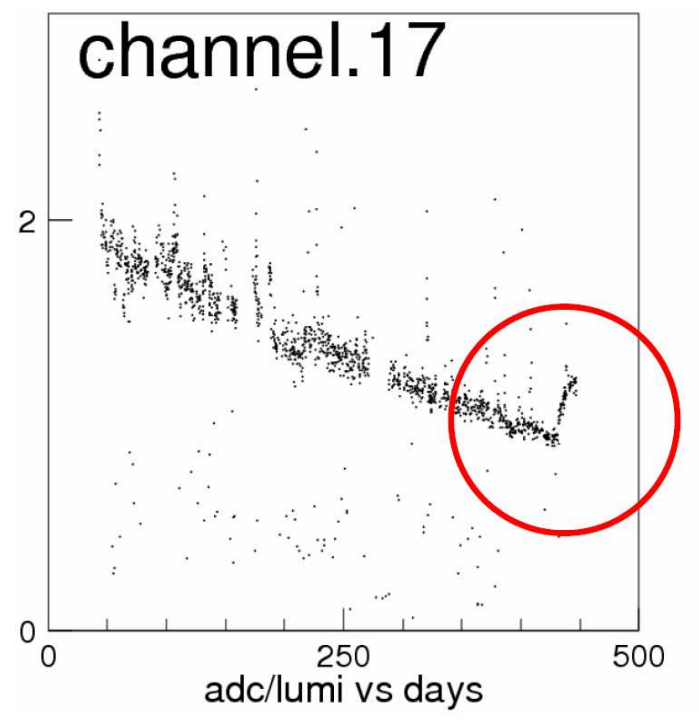

Figure 2.3 : The plot shows the average charge collected for a single channel normalized by the instantaneous luminosity plotted against time in days. Normalizing the charge values by luminosity reduces the variation in charge output due to multiple particles hitting a single wedge as a result of multiple interactions at higher luminosity. The gain drops with time due to radiation damage to the scintillator. The dry air purge began around day 425 and an increase in gain due to oxygen annealing can be seen circled in red. (from Y. Enari)

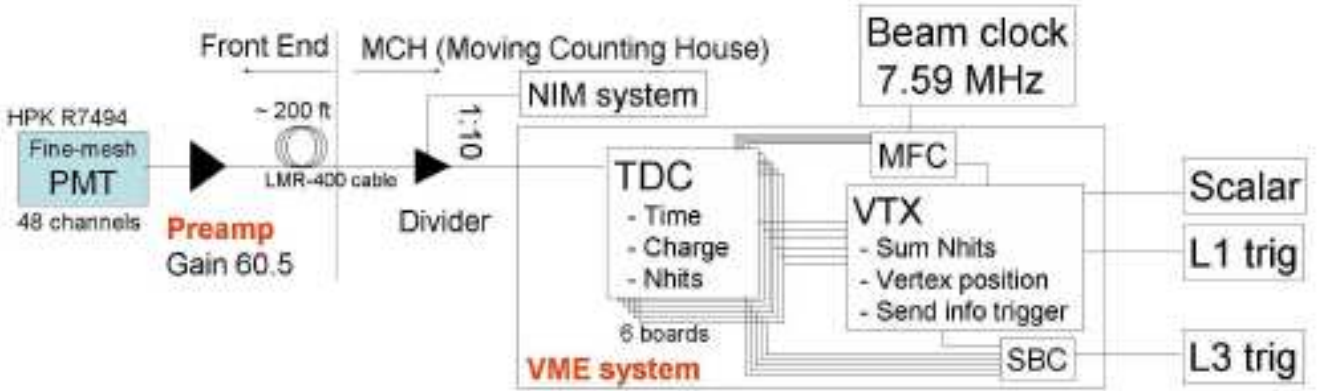

Figure 2.4: A schematic of the luminosity readout system. Analog signal from the PMTs in the luminosity monitor travel via cables to readout crates in the MCH. The signal is split between a NIM system, which makes the halo measurement, and a VME system, which makes the luminosity measurement. Luminosity information is sent to the Trigger Framework to be stored as scalars [10]. 


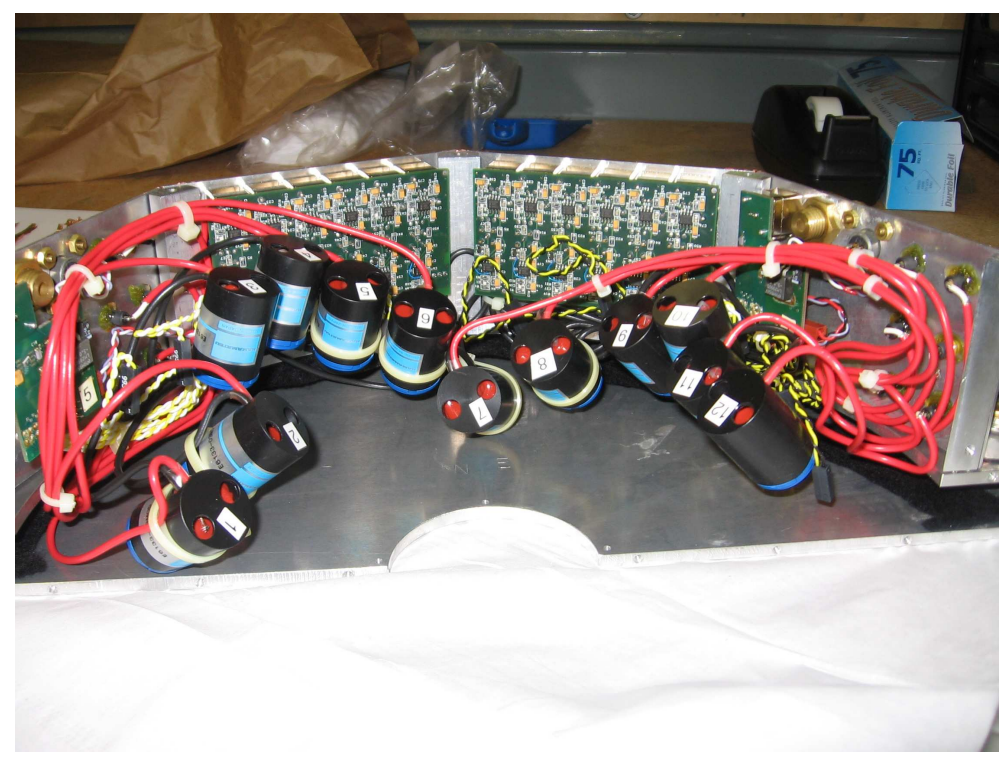

Figure 2.5 : A picture of the Northeast luminosity enclosure opened during a maintenance period in 2009 with the scintillator and PMTs removed. The two green boards in the center of the image are the pre-amplifier boards.

that can hit the luminosity monitor. These hits are early in one array of the luminosity monitor, but can be in-time hits in the downstream luminosity monitor array. Beam halo can affect the luminosity measurement and can damage systems near the beam pipe, like the SMT and Forward Muon system, so it is important to measure this value. Halo is measured as a hit rate and determined separately for the proton (North) and anti-proton (South) beams. The NIM electronics measure the halo rate by summing the analog charge signal seperately for the North and South arrays for each beam crossing and, if the charge passes the discriminator, comparing the difference in time between North and South. If the timing difference is sufficiently small, then the hits are in-time and used to make a NIM luminosity measurement. If the North (South) 
side is much earlier, then the crossing is declared to be a proton (anti-proton) halo hit, and therefore not used in the luminosity measurement. For normal running conditions the halo rates are below $15 \mathrm{kHz}$ for $p$ and $8 \mathrm{kHz}$ for $\bar{p}$.

The VME electronics are used for the official luminosity calculation. The VME readout uses an analog-to-digital converter (ADC) and a time-to-digital converter (TDC) so that both the charge and time information are collected for each channel. The slew-corrected time and charge information is sent to the VTX board where the data is sent to an online monitoring program called lmExamine, described in Section 2.3.2, as well as being used to determine the luminosity measurement. The VTX board makes timing and threshold cuts on each channel, determines if the halo veto is passed, and determines if a North/South coincidence occurred. The timing cut for a signal to be in-time is $\pm 6.4 \mathrm{~ns}$, which is a $3.5 \sigma$ window for the signal timing distribution. The threshold cut is equivalent to $30 \mathrm{mV}$ or about half of the minimum ionizing particle signal, which is 5 times greater than the electronics noise. The VME system also monitors halo rates. If more than 6 early hits occur in either side of the luminosity monitor in the VME calculation, that beam crossing will fire the halo veto and not be used to make the luminosity measurement. This decreases the possibility of an in-time hit not being seen due to the early hits since only one timing calculation is made for each channel. After applying these criteria, the VTX board makes the luminosity determination.

All appropriate information from the NIM electronics is collected by the VTX 
board in the VME system and the measurements from both systems are sent to the Trigger Framework (TFW). The TFW collects and tracks all luminosity related information, including luminosity, halo, and live time for the VME and NIM electronics, for each bunch crossing in the form of scalars and sends the scalar values out to be recorded with the rest of the $\mathrm{D} \varnothing$ detector information. Luminosity information is collected in time chunks, normally about a minute, that are designated by Luminosity Block Number (LBN) and stored in the online system, offline files, and in the luminosity data-base.

\subsection{Operations}

As with any detector, the system must be properly maintained to keep it running at peak efficiency. Care is taken to keep the DØ luminosity measurement within a $0.5 \%$ stability range. Most of the operations work for the luminosity system consists of keeping the calibrations up to date and working to improve the robustness of the system.

\subsubsection{Calibrations}

One benefit of upgrading from the NIM system to the VME electronics system is the ability to read out all channels individually. This in turn allows for single channel calibrations of timing, ADC pedestals, and gains. The ADC pedestals are checked monthly because they can drift with time and temperature. A calibration is performed 
every two to three months, as needed, to keep the pedestals within a $\pm 4 \mathrm{pC}$ range. The timing signal from the Accelerator Division changes 1 to 2 ns with the seasonal temperature variations, which is noticeable on the timescales used at DØ. An accurate timing calibration keeps the signal for data within the in-time cuts used to exclude halo hits from the luminosity measurement. The timing calibration is changed four to six times a year but is checked almost daily.

As the scintillator is hit with radiation it degrades, and the amount of light output begins to diminish. To combat this effect and keep the acceptances on the detector stable, the high voltages on the PMTs are periodically raised. The high voltage (HV) of the PMTs are individually set so that the anode current is equivalent to $18 \mu \mathrm{A}$ at an instantaneous luminosity of $300 \mathrm{E} 30 \mathrm{~cm}^{-2} \mathrm{~s}^{-1}$, which is a gain of approximately $10^{4}$ in the PMT.

\subsubsection{Monitoring}

One important monitoring tool is the online program lmExamine. The program samples the readout from the luminosity monitor during data taking using the zero-bias trigger from the $\mathrm{D} \emptyset$ data acquisition system. This means that lmExamine includes information from data and empty ticks. LMExamine tracks the timing and charge

output for each channel, an example of which can be seen in Figure 2.6. The examine creates a new Paw [11] file, or NTuple, for each run, which is a division in data taking. Along with timing and charge distributions during data taking, lmExamine is used 
to check the ADC pedestals which must be done when no beam is in the Tevatron.

The lmExamine program is also helpful in monitoring the gains of the channels to determine if a $\mathrm{HV}$ update is needed. Information from lmExamine is compiled to produce a plot of the average ADC count per instantaneous luminosity versus integrated delivered luminosity for each channel, as shown in Figure 2.7.

Information from the ADC versus luminosity plots is combined with $\mathrm{HV}$ and threshold scans to check the stability of the gains. HV and threshold scans are done about once a month. Figure 2.8 shows results from a selection of HV scans, and Figure 2.9 shows the output from threshold scans. The scans and plots are used to determine if the HV settings need to be updated.

Another piece of monitoring is done through direct communication with the VME crate. A network connection, telnet, allows communication with the VTX board through a program called VXWorks. The connection to the VTX board is necessary for processes such as changing the threshold cut during threshold scans and downloading new timing and ADC pedestal calibrations. One feature of the system is the ability to retrieve data directly from the VTX board, including the hit count of the North and South luminosity monitor arrays, which was very useful in the determination of the Run IIB luminosity constant. 

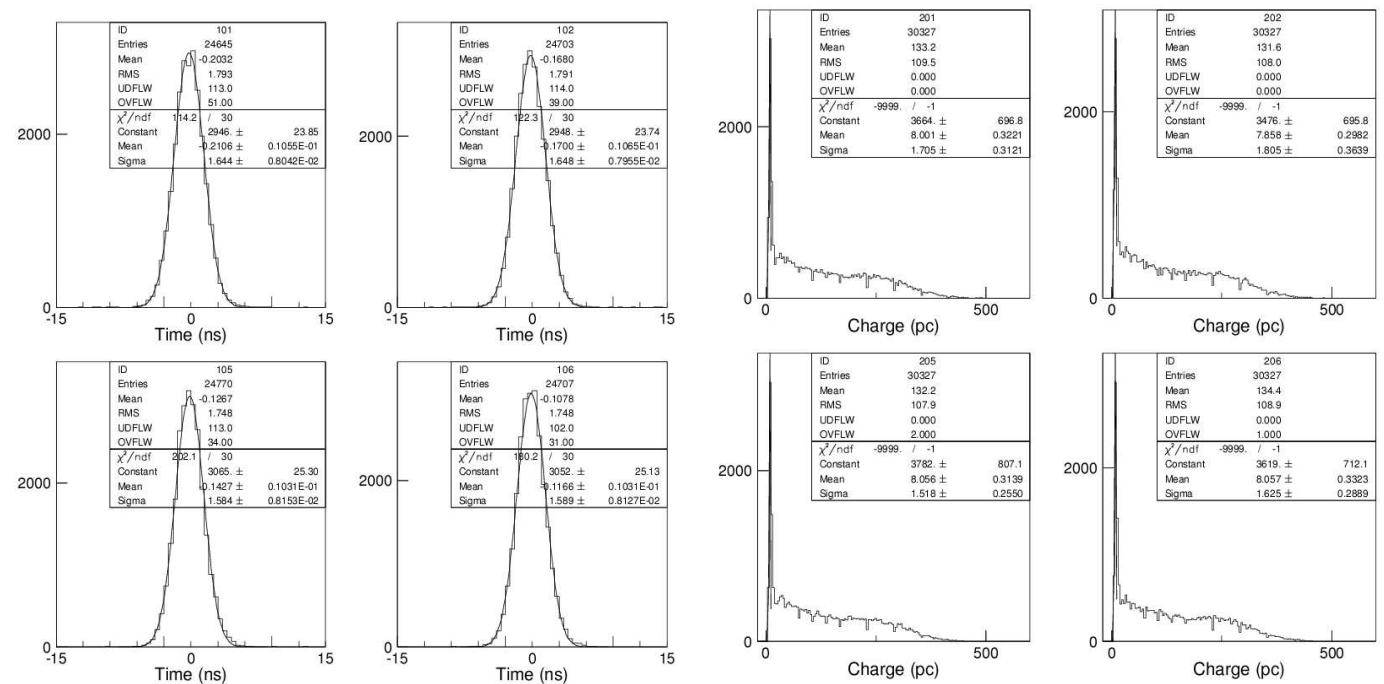

(a) Timing (ns)

(b) Charge (pC)

Figure 2.6 : Example of timing and charge distributions from lmExamine for four channels during Run 259781 on March 22, 2010, over a luminosity range of 100 - 70E30.

\subsubsection{Maintenance}

Over the past two years of running, the HV was raised after approximately every $1 \mathrm{fb}^{-1}$ of integrated delivered luminosity. When the combination of information from the ADC versus luminosity plots and HV and threshold scans show that the HV on the PMTs needs to be increased, special runs need to be taken for at least three different HV values. Normally one run is taken with the HV set around the current value, and the other two runs are taken with different settings above the current set point. After the special runs are completed, the charge information for each channel is taken from the lmExamine file. The data is compiled to give the gain versus HV plot, and a linear 


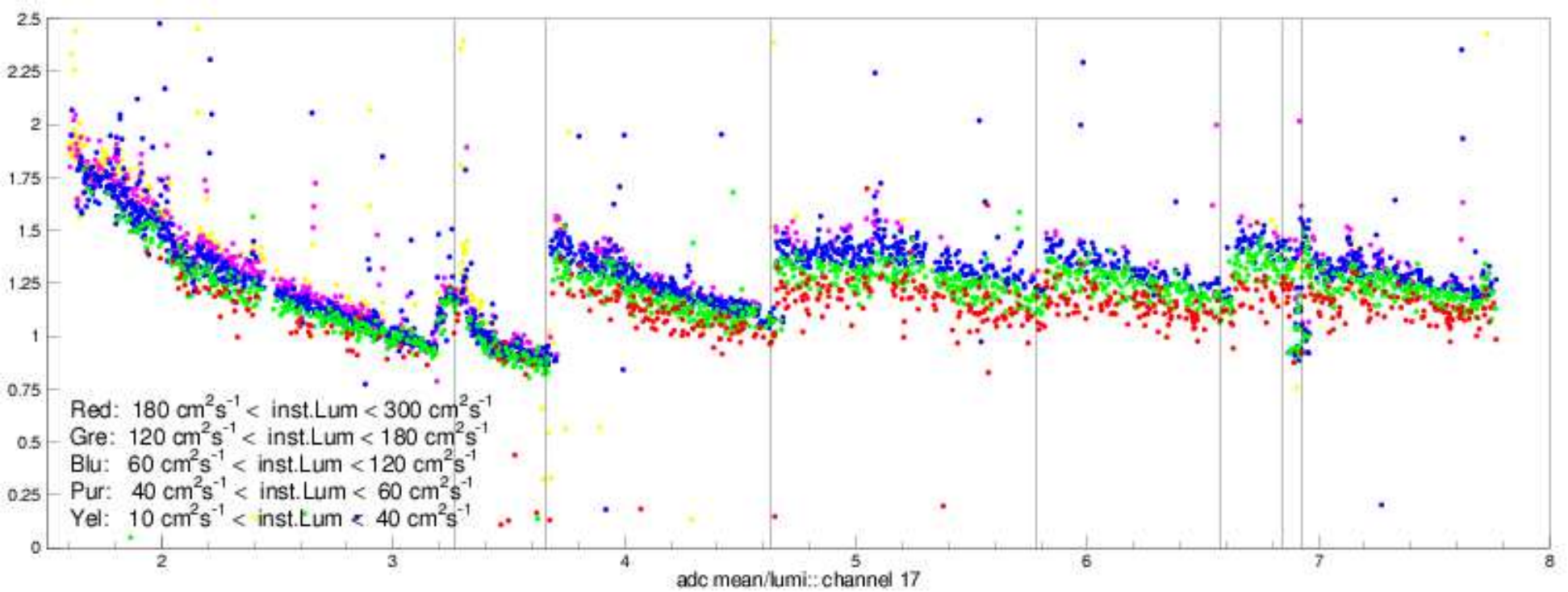

Figure 2.7 : Plot of the average ADC values normalized by the instantaneous luminosity (in arbitrary units) versus the integrated delivered luminosity $\left(\mathrm{fb}^{-1}\right)$. Each point represents a different data-taking run. The vertical lines denote HV updates or scintillator changes, which were necessary to keep the normalized ADC values above 1. 


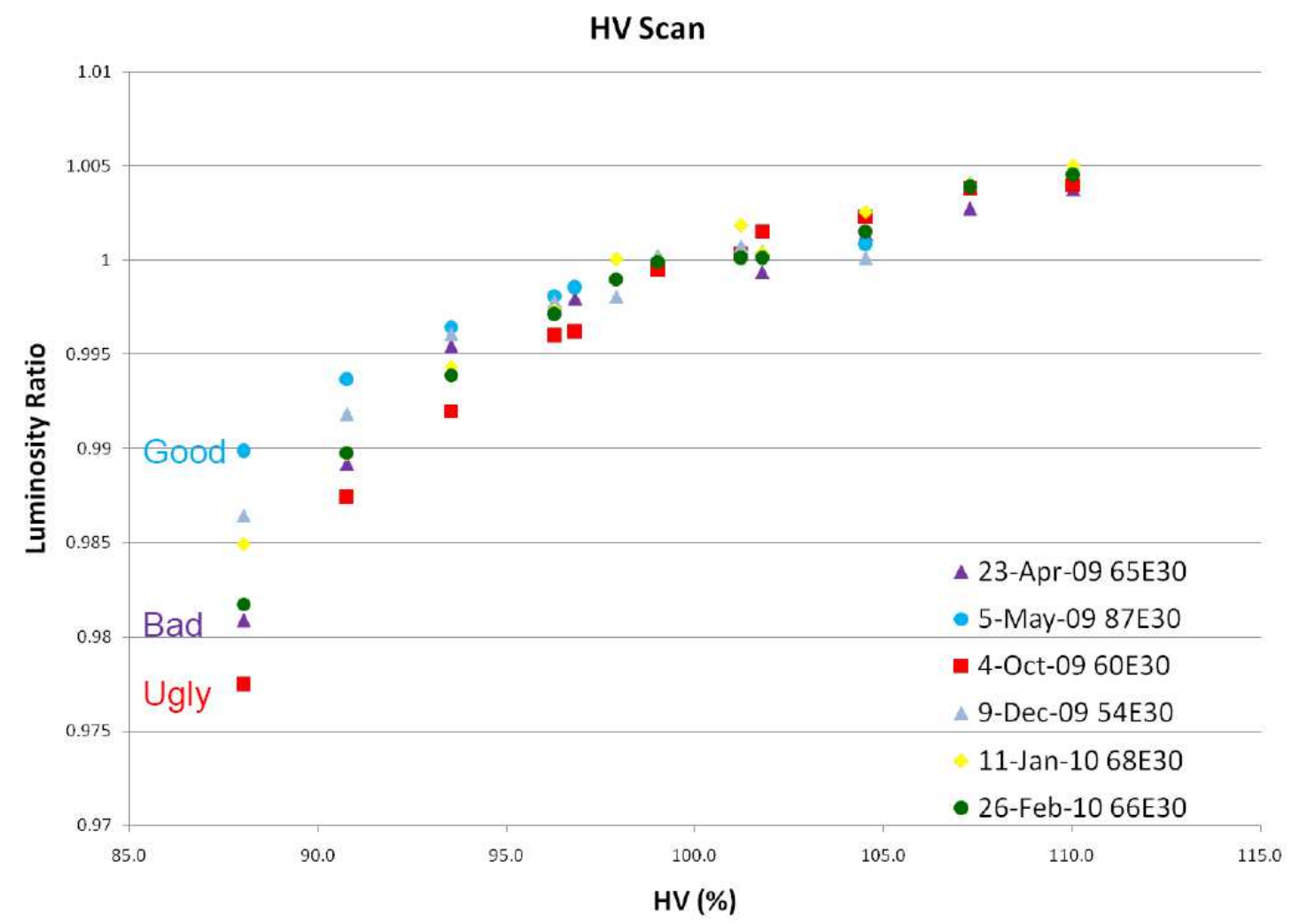

Figure 2.8 : Plot of HV scan results. The operational set point of the HV is at $100 \%$. If the luminosity ratio falls sharply below the $100 \% \mathrm{HV}$ value, as with the $23-\mathrm{Apr}-09$ scan, it is time to raise the HV. The 5-May-09 scan is an example of a good HV setting. 
Threshold Scan

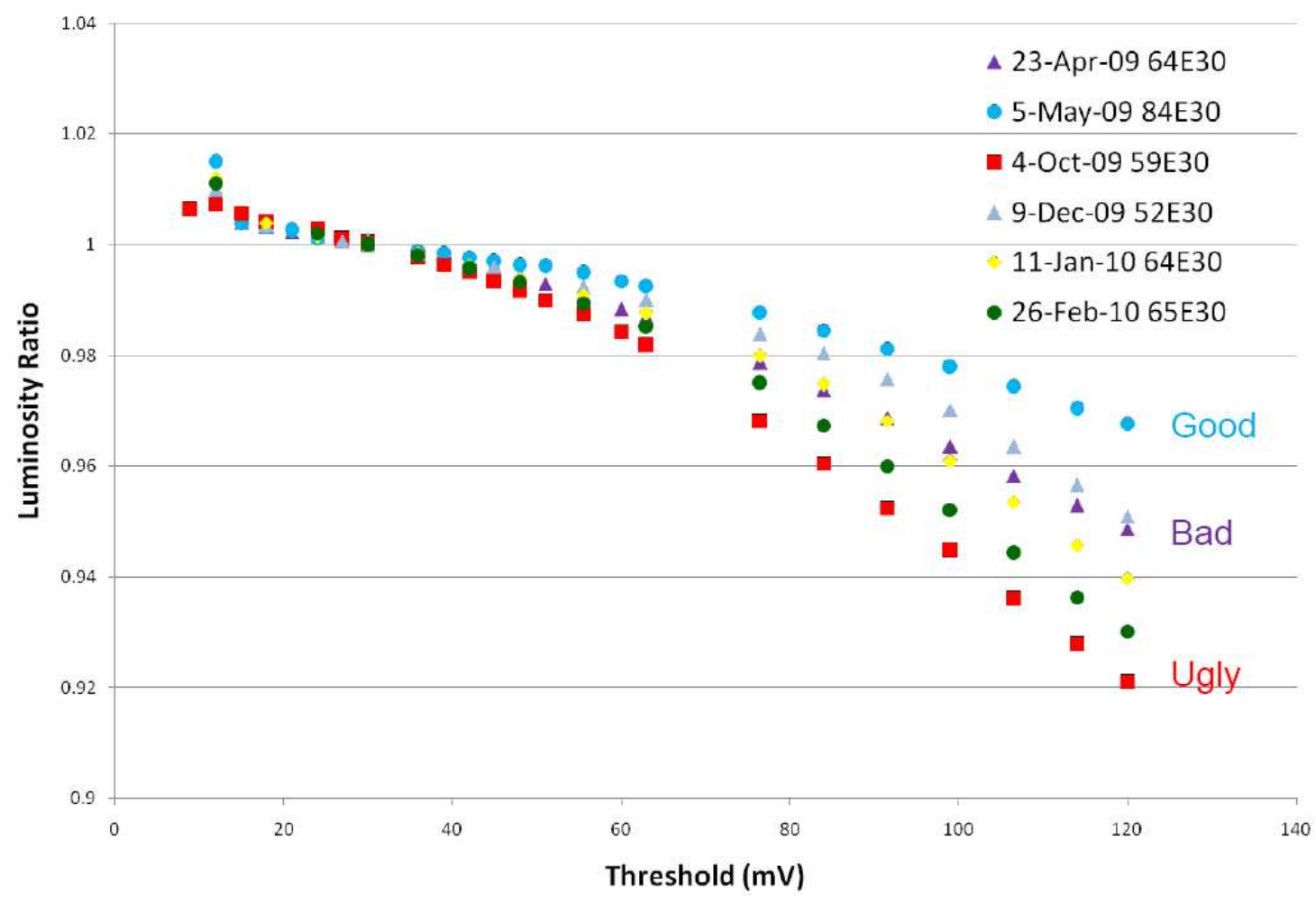

Figure 2.9 : Plot of threshold scan results. The threshold cut for normal operations is set at $30 \mathrm{mV}$. The scan on 5-May-09 show that the HV settings are good, while the 23-Apr-09 scan indicate that the HV needs to be raised. 
fit is used to determine the $\mathrm{HV}$ value for each channel that corresponds to the correct gain setting. Testing is done before the settings are made official to confirm that the luminosity is still within the expected $0.5 \%$ stability range. Figure 2.10 shows a single channel example of the charge distribution during each of the special runs and the fit done to determine the new HV setting.

Keeping the gains stable by increasing the HV on the PMTs only works if the scintillator is not severely depleted and the maximum voltage on the PMTs has not been reached. Therefore, when the opportunity of a long shutdown arises, the luminosity group operations team removes the luminosity monitor and replaces all of the scintillator. New scintillator was put into the detector in 2006 and changed again in the summers of 2007 and 2009. A visual comparison of the radiation damaged scintillator to a new piece of scintillator is shown in Figure 2.11. During these long down times, the PMTs can also be replaced if necessary. During the shutdown in 2006, 4 of the 48 PMTs were replaced. The plan is to change the scintillator again in the summer of 2010. During this shutdown, replacement PMTs will also be put in for channels that are predicted to exceed the maximum allowed HV setting before the end of Tevatron running. Overall, the luminosity monitor is a robust detector that is extremely important to physics research at $\mathrm{D} \varnothing$. 

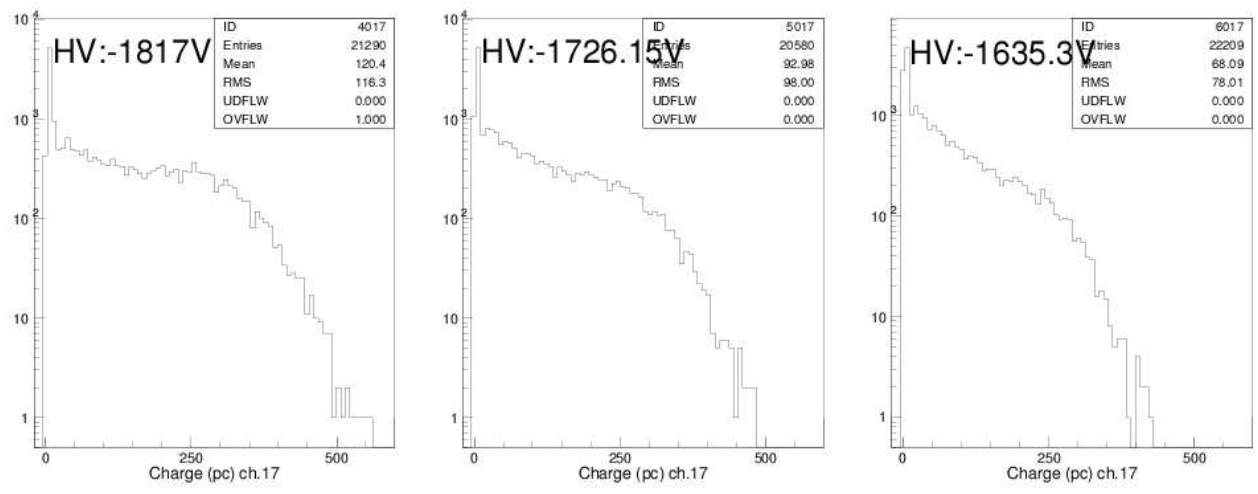

(a) Charge (pC)

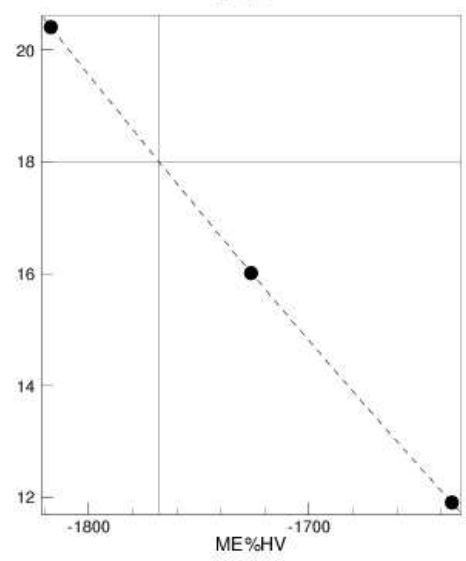

(b) Annode Current $(\mu \mathrm{A})$ vs. HV (V)

Figure 2.10 : Plot of the charge output for the three special runs and the linear fit used to determine the HV setting for a gain that corresponds to a PMT annode current of $18 \mu \mathrm{A}$ at an instantaneous luminosity of $300 \mathrm{E} 30 \mathrm{~cm}^{-2} \mathrm{~s}^{-1}$. 


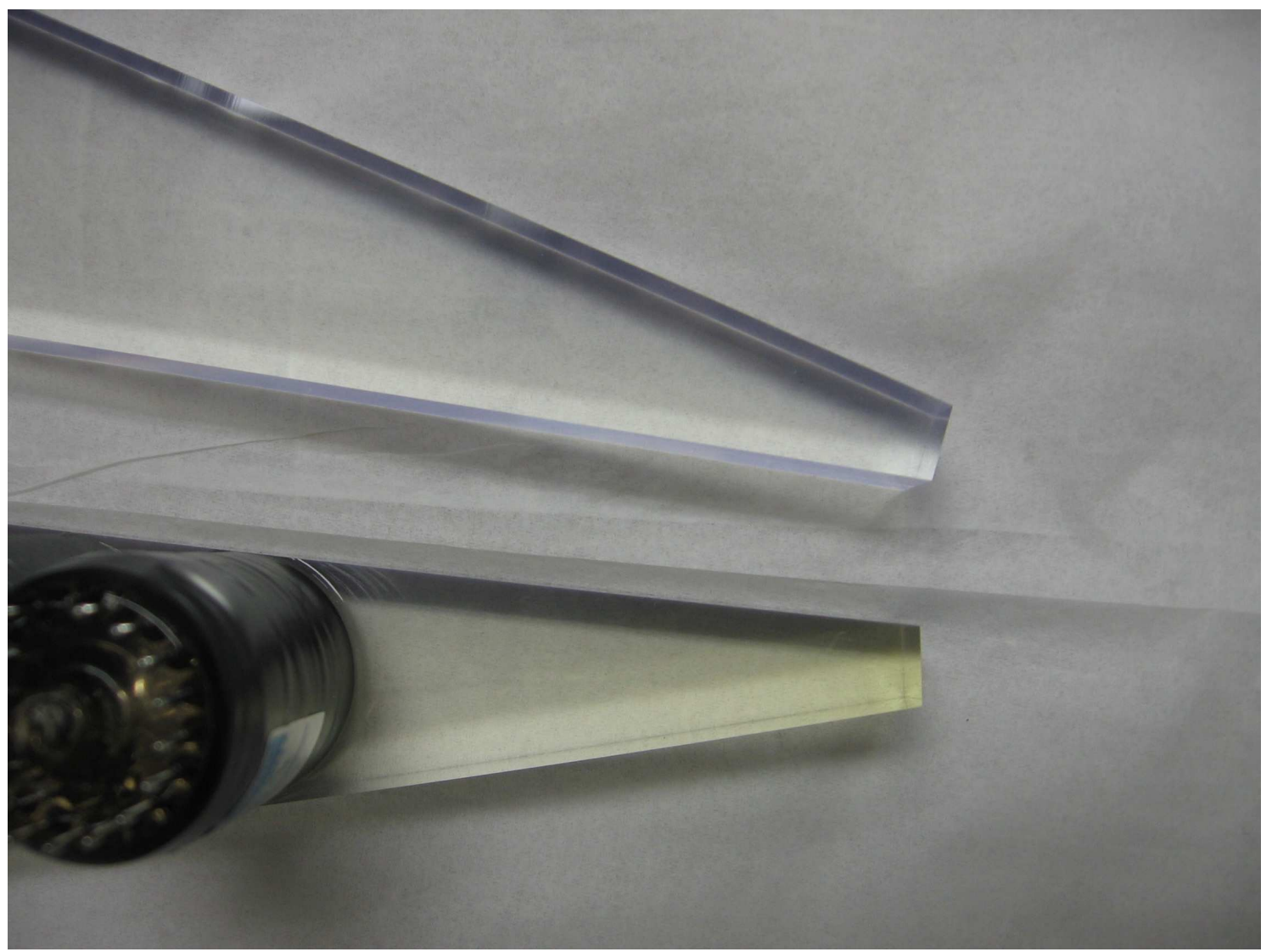

Figure 2.11 : The visible yellowing at the inner region of the lower scintillator wedge is due to radiation damage of the wedge. The upper wedge is a new piece of scintillator that was put in during a maintenance period to replace the damaged piece. 


\section{Chapter 3}

\section{Run IIB Luminosity Constant}

\subsection{Luminosity Measurement}

The technique for measuring luminosity at DØ is referred to as "counting empties," because the luminosity is calculated from the rate of empty crossings. For the purpose of the luminosity calculation, an empty crossing is defined as a bunch crossing in which an in-time coincidence in both the North and South sides of the luminosity monitor does not occur. Each bunch is unique, having different numbers of protons and anti-protons. For this reason, the luminosity is calculated separately for each bunch. Using Poisson statistics, the relationship between the probability of an empty crossing, $P(0)$, and the luminosity, $L$, is given in Equation 3.1.

$$
P(0)=e^{-\sigma_{e f f} L / \nu} *\left(2 e^{\left(-\sigma_{S S} / 2 \nu\right) L}-e^{-\sigma_{S S} L / \nu}\right)
$$

Here $\sigma_{e f f}$ is the effective inelastic cross section seen by the luminosity monitor, $\nu$ is the crossing frequency for the bunch, and $\sigma_{S S}$ is the single-sided cross section. The crossing frequency for a bunch is about $47 \mathrm{kHz}$. The single-sided cross section is the cross section for firing only one array, North or South. The factor in parenthesis is a 
correction for two single-sided interactions overlapping and hitting both sides of the monitor, thus faking a double-sided event. The effective inelastic cross section for the luminosity monitor, $\sigma_{e f f}$, is often referred to as the luminosity constant. To be able to accurately determine the luminosity, both $\sigma_{S S}$ and $\sigma_{\text {eff }}$ must be determined.

The single-sided cross section, given in Equation 3.2, is the combination of the fraction of each of the three types of inelastic collisions, non-diffractive (ND), single diffractive (SD), and double diffractive (DD), discussed in Section 1.5, and the singlesided acceptances for each type.

$$
\sigma_{S S}=\sigma_{I N} *\left(f_{N D} * A_{N D}^{S S}+f_{S D} * A_{S D}^{S S}+f_{D D} * A_{D D}^{S S}\right)
$$

In this equation, $\sigma_{I N}$ is the total inelastic cross section, $f$ represents the fraction of events of the type denoted in subscript and $A$ represents the acceptance for that event type in a single side (SS), which is a combination of North only (NO) or South only (SO) hits. The determination of the acceptances and fractions will be explained in the following sections. In practice, the single-sided cross section also includes a background piece. The background correction is not a true cross section but does behave as an effective cross section, since it varies linearly with luminosity. More information on the current luminosity background correction can be found in DØNote $5946[12]$. 


\subsection{Luminosity Constant}

The determination of the Run IIB luminosity constant is crucial to the physics analyses at DØ. During the shutdown in 2006, which separates the Run IIA and Run IIB periods at $\mathrm{D} \varnothing$, a new detector layer was added to the silicon subsystem. The addition of this material and changes to components in the forward region to allow for the installation meant that the luminosity constant would need to be re-determined for the Run IIB period. For more information on the calculation of the Run IIA luminosity constant see DØNote 4958 [7].

The luminosity constant is calculated with information from previous experiments, data, and Monte Carlo (MC) simulations. It can be simply written as the combination of the acceptances, $A$, and the cross sections, $\sigma$, for each of the three types of inelastic collisions, as shown in Equation 3.3.

$$
\sigma_{e f f}=\sigma_{N D} * A_{N D}+\sigma_{S D} * A_{S D}+\sigma_{D D} * A_{D D}
$$

The acceptances for each event type are determined from MC simulations. Using a given MC simulation sample, the acceptance is calculated as the fraction of events in which at least one particle hits the detector, depositing enough in-time energy to pass the timing and threshold cuts. Acceptances are determined separately for North only, South only, and a coincidence in North and South, as can be seen in the example in Table 3.1. 


\begin{tabular}{|c|c|c|c|c|}
\hline Type & North + South & North only & South only & Neither N or S \\
\hline ND & $0.988 \pm 0.001$ & $0.005 \pm 0.001$ & $0.007 \pm 0.001$ & 0 \\
SD & $0.319 \pm 0.006$ & $0.226 \pm 0.005$ & $0.227 \pm 0.005$ & $0.228 \pm 0.005$ \\
DD & $0.479 \pm 0.006$ & $0.223 \pm 0.005$ & $0.209 \pm 0.005$ & $0.090 \pm 0.004$ \\
\hline
\end{tabular}

Table 3.1 : Acceptances for the preliminary Run IIB geometry for each type of inelastic event, non-diffractive (ND), single diffractive (SD), and double diffractive (DD). The acceptances were recalculated for all changes to the Monte Carlo simulation [13].

The cross sections for each type of event, given in Equation 3.5, are defined as the fraction of those types of events multiplied by the total inelastic cross section, $\sigma_{I N}$.

$$
\begin{aligned}
\sigma_{N D} & =f_{N D} * \sigma_{I N} \\
\sigma_{S D} & =f_{S D} * \sigma_{I N} \\
\sigma_{D D} & =f_{D D} * \sigma_{I N}
\end{aligned}
$$

The total inelastic cross section at Tevatron Run II energy used by both D $\varnothing$ and $\mathrm{CDF}$ is $\sigma_{I N}=60.7 \pm 2.4 \mathrm{mb}$. The inelastic cross section was measured at an energy of $\sqrt{(} s)=1.8 \mathrm{TeV}$ by experiments at CDF [14], E710 [15], and E811 [16]. The results were combined and extrapolated to an energy of $980 \mathrm{GeV}$ per beam. The fraction of non-diffractive events must be determined by comparison of the data and MC. The fraction of single diffractive to total diffractive events is taken from other experiments and is given in Equation 3.5 [7].

$$
f_{S D} /\left(f_{S D}+f_{D D}\right)=0.57 \pm 0.21
$$


In the determination of the luminosity constant this ratio is varied over a large, but reasonable range, and the change to the constant was taken as part of the systematic uncertainty. More information about the uncertainties can be found in Section 3.7.

\subsection{Monte Carlo Simulation Samples}

The technique used to determine the luminosity constant required several MC simulation samples. Sample sets were generated separately for non-diffractive (ND), single diffractive (SD), and double diffractive (DD) event types. The generator for the samples is Pythia [17] version 6.409 with the Tune A [18] parameter settings. Particles are then tracked through the $\mathrm{D} \varnothing$ detector using d0gstar, which is a $\mathrm{D} \varnothing$ supported detector simulator based on GEANT 3 [19]. The output from d0gstar is passed through d0sim, which takes the raw signal from d0gstar and simulates the signal as it would be read out from the $\mathrm{D} \varnothing$ detector. D0reco then reconstructs the detector readout into physics data, as is done when processing real data from the detector. After the entire chain is followed, the MC simulation sample sets of each type are complete. Then events must be overlaid to make sample sets that accurately represent the data at a specific luminosity.

\subsubsection{Run IIB Upgrades to the Monte Carlo Simulation}

Before the effective cross section for the luminosity monitor could be determined for Run IIB data, the geometry model in the MC simulation needed to be updated for the 
new material. The main change to the D $\varnothing$ detector between Run IIA and Run IIB was the addition of a layer to the silicon detector called Layer 0 . Layer 0 consists of a new set of silicon sensors that sits between the beam pipe and the other barrel layers of the silicon detector. Layer 0 also included cable readout and mechanical support structure. In order to fit the new material into the existing detector, the beam pipe used during Run IIA had to be replaced with a smaller diameter pipe. The smaller beam pipe required the addition of support material on the pipe including new flanges and bellows. Cables were added in the forward region to allow readout from Layer 0. Since the readout for the Silicon detector was already at full capacity, one H-disk, see Figure 3.1, from each side of the detector was removed to allow the new readout. This also meant that new thermal and structural support was added to the area where the detector material was removed. In all, new sensor, structural, and electrical material was added with the addition of Layer 0 .

The changes to the sensor material were added to the standard D $\varnothing \mathrm{MC}$ geometry

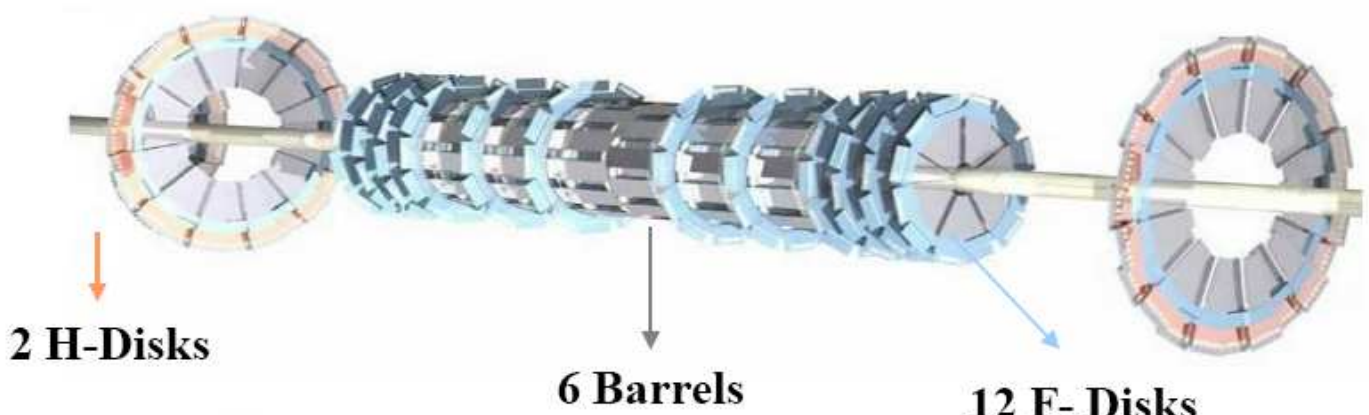

Figure 3.1 : Drawing of the Run IIB silicon microstrip detector sensors. The Run IIA silicon detector did not included Layer 0 but had $4 \mathrm{H}$-disks in total. (from S. Desai) 
package for analysis purposes. However, it was necessary to add all the mechanical and electrical support material in the forward region to a special version of the MC simulation used by the luminosity group since it changed the amount of material seen by particles heading toward the luminosity monitors and had an impact on the total multiplicity observed in the luminosity monitor. Figure 3.2 and Figure 3.3 compare the standard DØ MC geometry to the upgraded geometry used for this analysis.

Another upgrade to the $\mathrm{D} \varnothing$ material model was in the luminosity system itself. Upgraded pre-amplifier readout boards were put into the luminosity monitor during the shutdown before Run IIB. This material was added to the d0gstar model as well as a more detailed description of all the material inside the luminosity monitor enclosure. The improvements, shown in Figure 3.4, included material for the PMTs, the preamplifier boards, the signal and HV cables, and the aluminium support structure inside the enclosures.

In the Run IIA analysis, extensive work was done to tune d0sim to reproduce the correct light yield as a function of position of the initial particle hit. The work included cosmic ray studies, the results of which are shown in Figure 3.5. Details of the experiment can be found in DØNote 5255 [20], and more information on the d0sim tune can be found in DØNote 4958 [7]. The type of PMT and scintillator used in the luminosity monitor was not changed between Run IIA and Run IIB, therefore it was not necessary to change this part of d0sim. Tuning was still done to accurately reflect the time and charge distributions seen in the data. For the timing, a slewing 


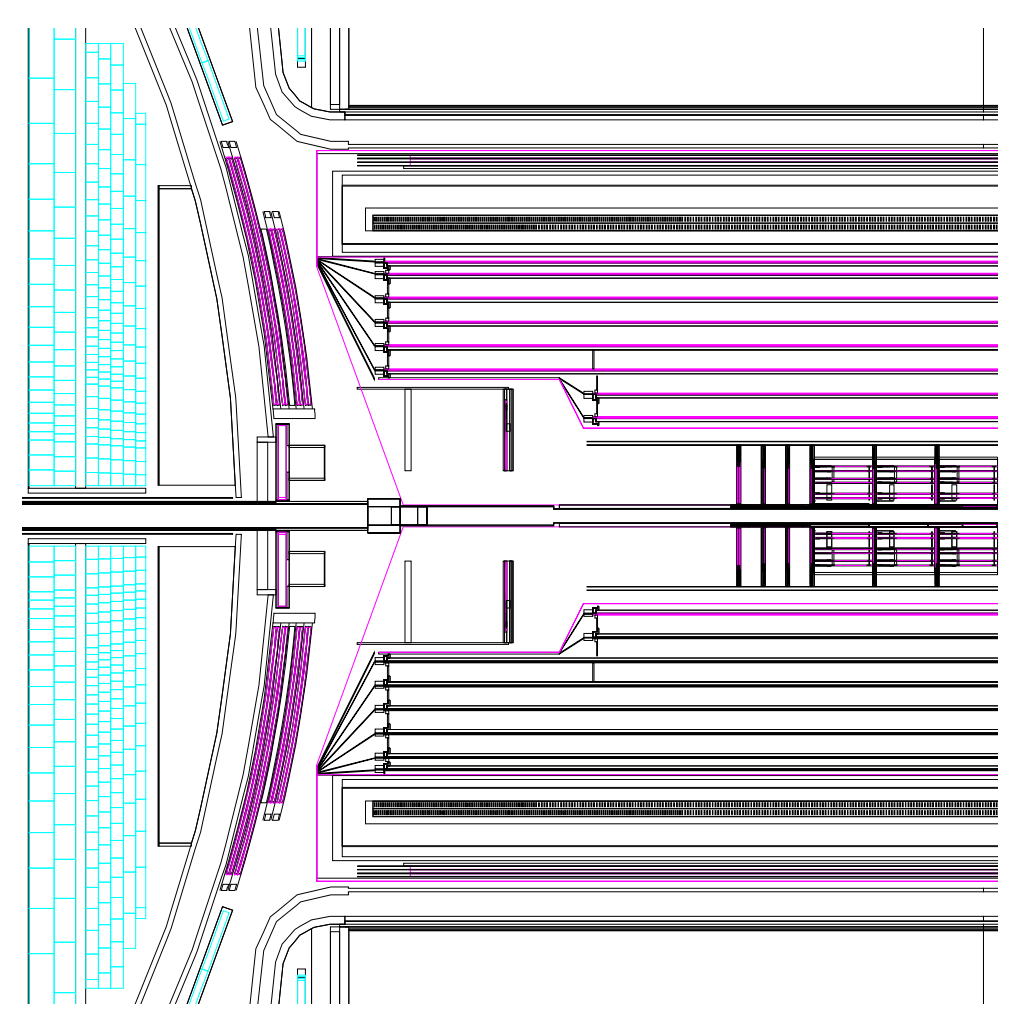

(a) Official Dø Geometry

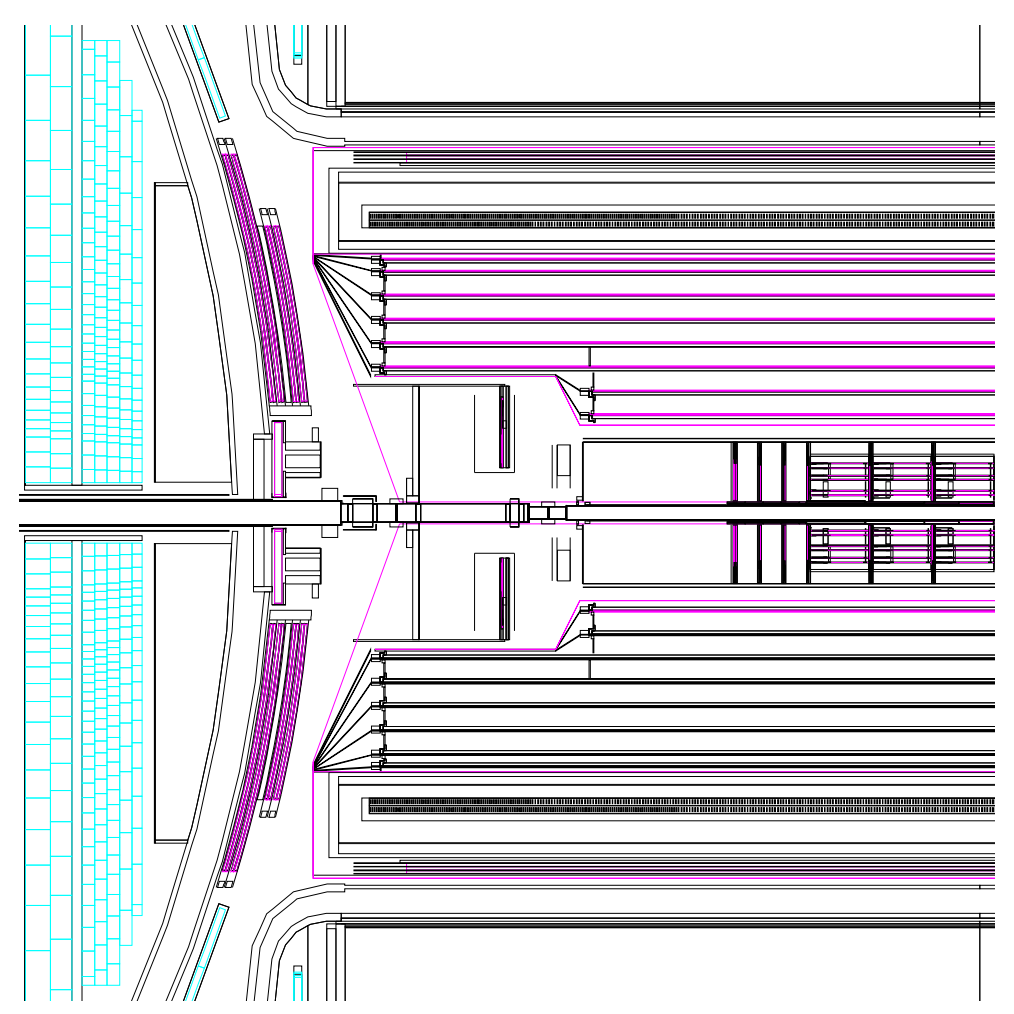

(b) Upgraded Geometry

Figure 3.2 : The half slice of the DØ Detector shows the tracking system, luminosity monitor, and end cap calorimeter. The addition of material to the forward region of the detector simulation is visible. 


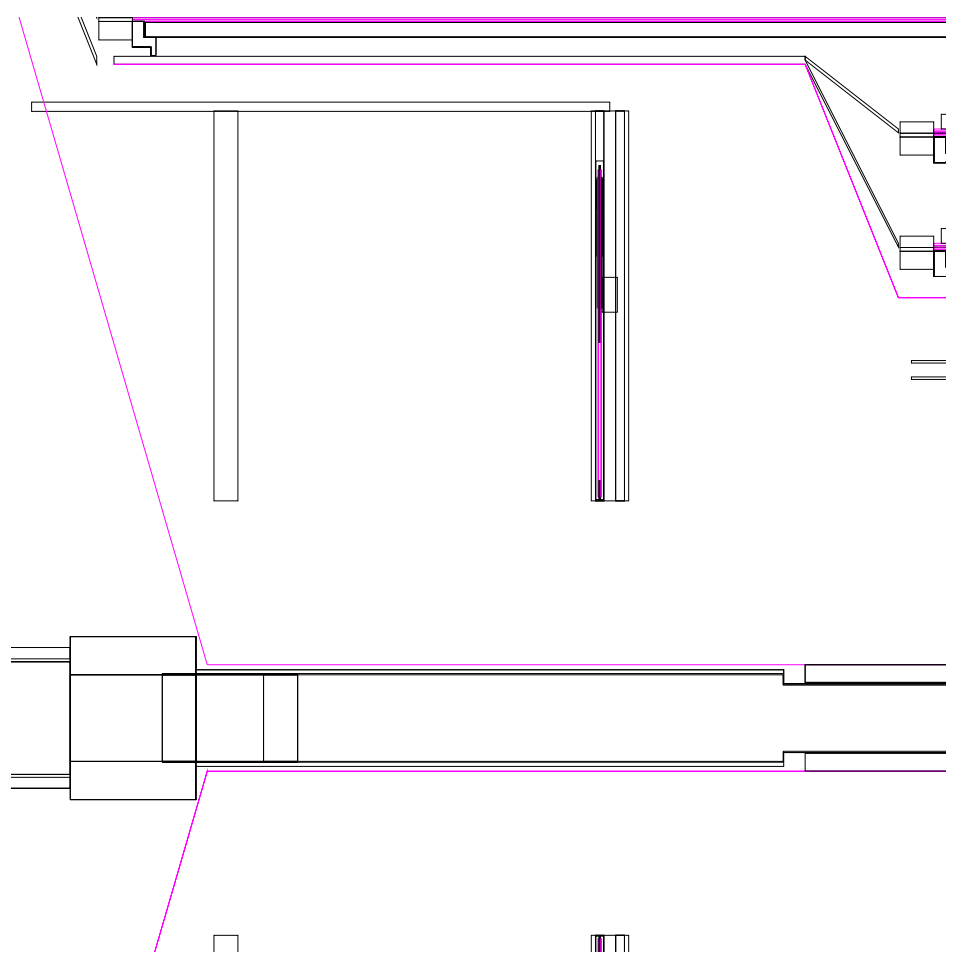

(a) Official D $\varnothing$ Geometry

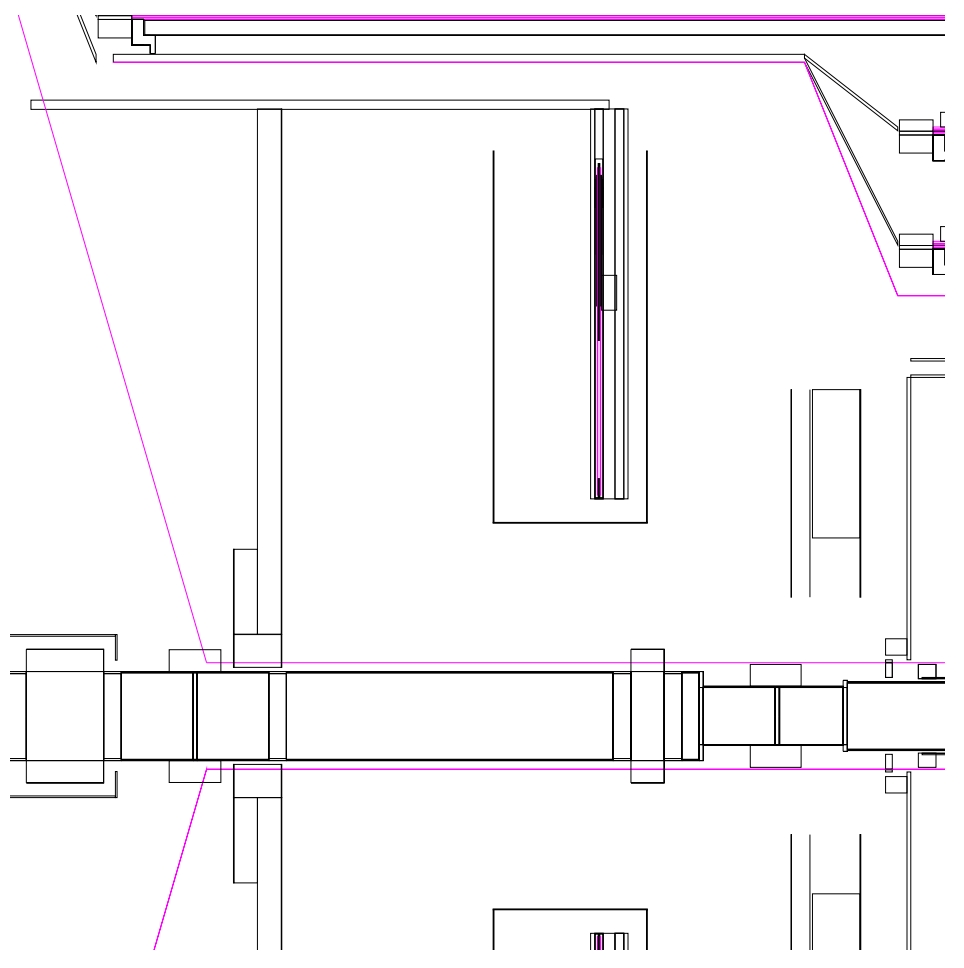

(b) Upgraded Geometry

Figure 3.3 : A zoomed in view of the beam pipe and H-disk region of the detector. The additional material shown includes flanges and bellows on the beam pipe, beam pipe support sturcture, and Layer 0 readout components. 

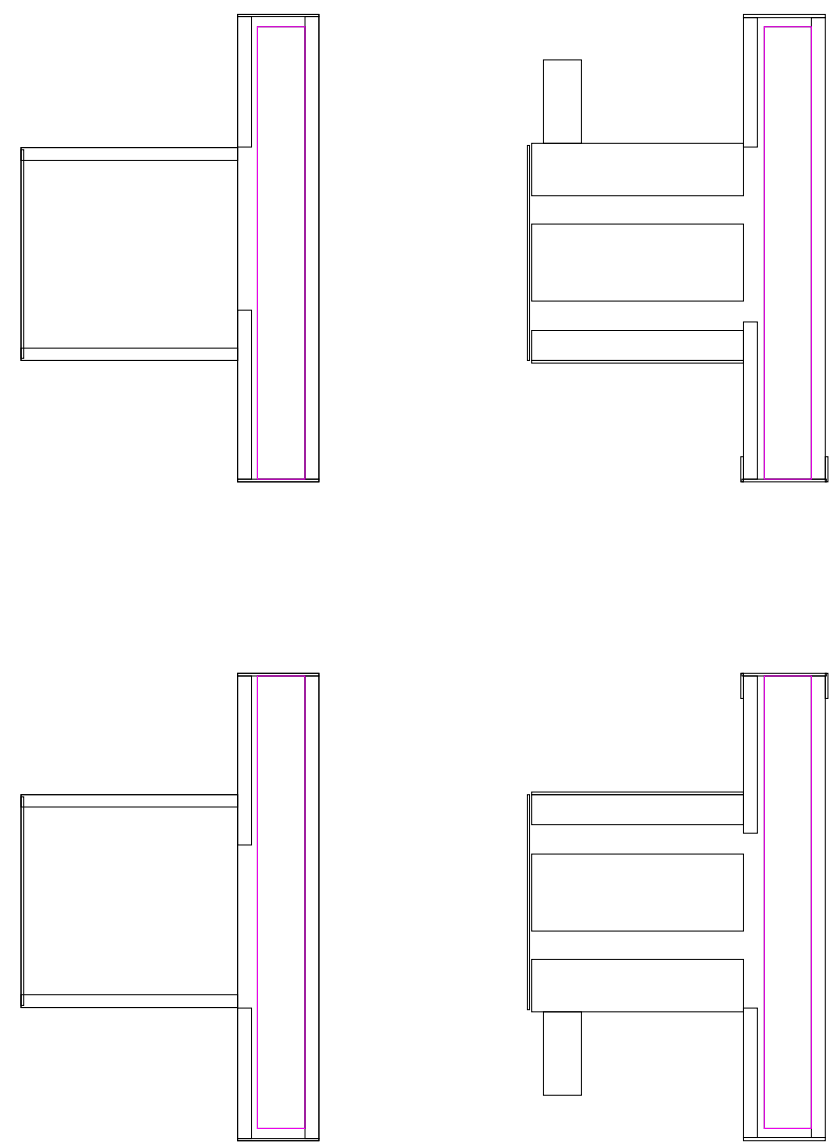

(a) Official DØ Geometry

(b) Upgraded Geometry

Figure 3.4 : A view of one array of the luminosity monitor cut perpendicular to the beam pipe. The pink line denotes the scintillator. The size and density of the aluminium was adjusted to more accurately represent the enclosure material. Material was also added for the PMTs inside the enclosure and the signal readout outside the enclosure. 
correction was applied to the MC simulation, as is done in the data, to correct for the offsets in time related to the charge of the hit. It was also important to replicate the width of the timing distribution so that the cuts of \pm 6.4 ns could be applied to the MC simulation. Figure 3.6(a) shows a single channel example of the comparison of the data and $\mathrm{MC}$ simulation timing distribution. To make an accurate comparison of the data and MC simulation charge distribution it was necessary to apply a corrected conversion of the energy deposited to the charge collected. As shown in Figure 3.6(b), the tuning aligned the minimum ionizing particle (MIP) peak placement in the data and MC simulation. The technique used to determine the location of the MIP peak included "consistently applying a complex neural network algorithm to an optically corrected retinal image sampled under controlled conditions," as described in DØNote 5945 [21]. This technique is also referred to as the eyeball method. After the tuning it was possible to make a threshold cut that is equivalent to the $30 \mathrm{mV}$ cut made in data to the MC simulation by using the appropriate fraction of the MIP peak [7].

\subsubsection{Monte Carlo Simulation Sample Overlays}

The luminosity measurement is made for each bunch crossing, so to be able to compare the MC simulation and the data, the MC simulation samples must simulate a bunch crossing. The number of each type of inelastic event in a bunch crossing depends on the luminosity and varies with Poisson statistics. To make a sample set that is comparable with data, events of each type must be overlaid. A different MC overlaid 

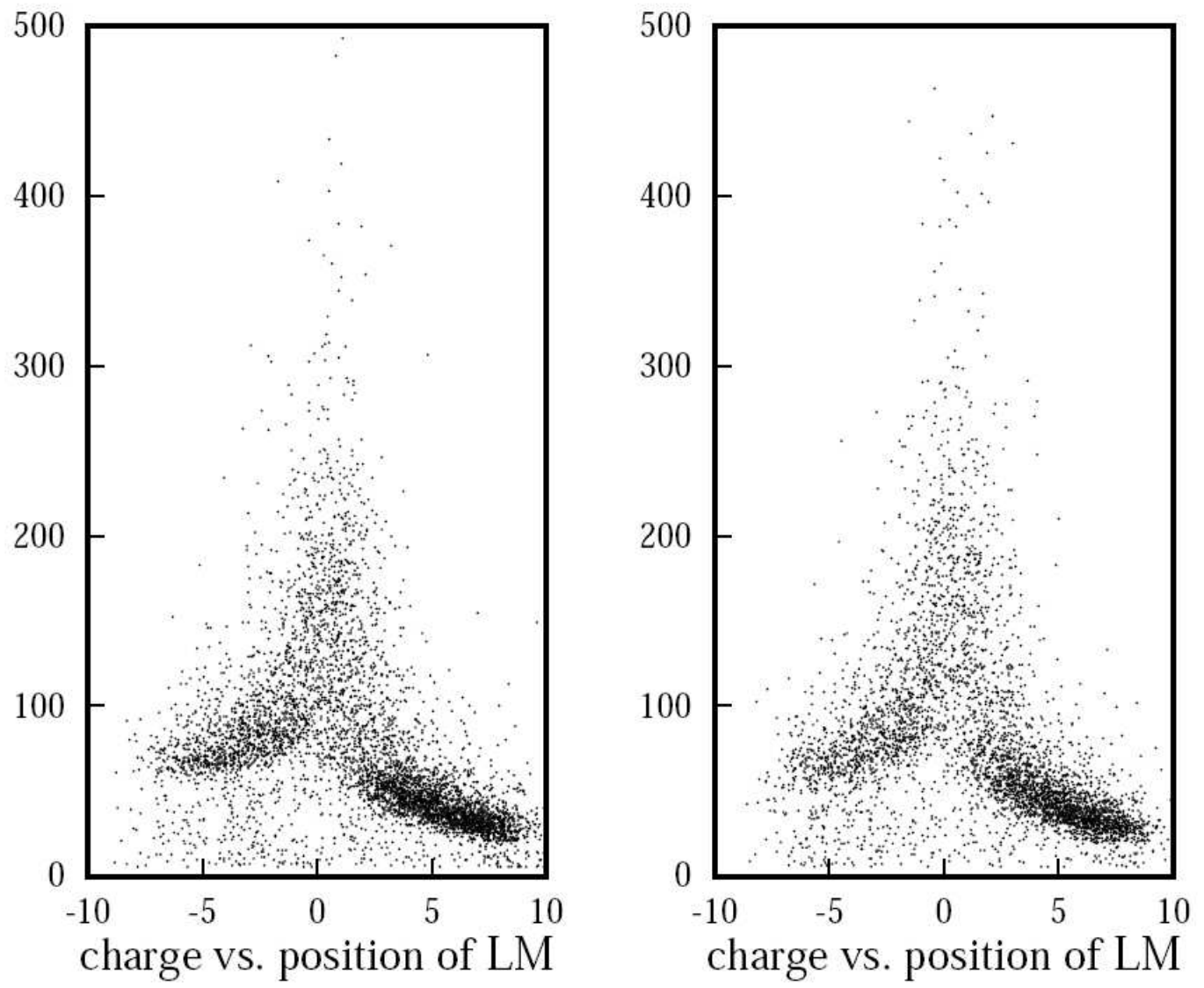

Figure 3.5 : Results of the cosmic ray studies to determine the relationship between particle hit position and light yield. The charge is read in $\mathrm{pC}$. The position is given in $\mathrm{cm}$ with $\mathrm{x}=0$ equivalent to a hit directly under the PMT and $\mathrm{x}=-6$ at the inner edge of the scintillator. The left plot was a new piece of scintillator, while the right plot is for an radiation damaged scintillator wedge. The results of this measurement were used to tune d0sim [20]. 


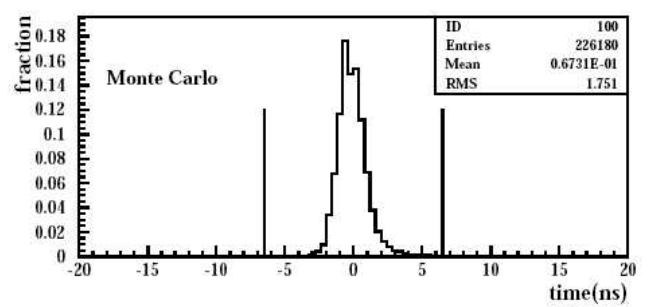

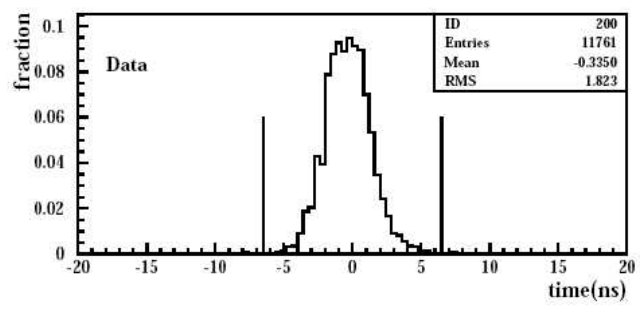

(a) Timing (ns)

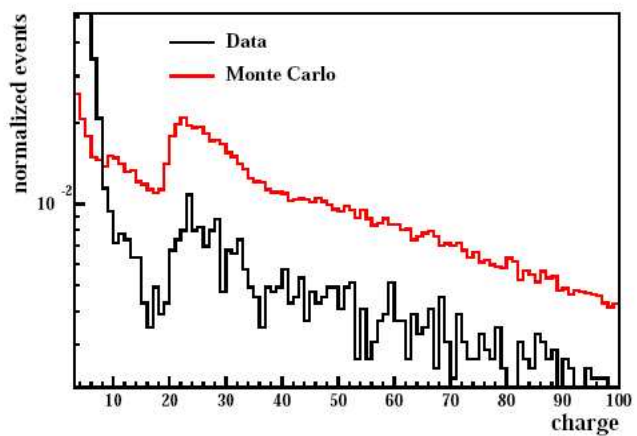

(b) Charge (pC)

Figure 3.6 : Comparison of the timing and charge distributions after tuning the MC simulation. The vertical lines in the timing plots correspond to the window for in-time hits [13].

sample has to be created for each value of the luminosity and the non-diffractive fraction, $f_{N D}$.

For a specific luminosity and $f_{N D}$, a MC simulation overlaid sample is created by determining the number of each type of interactions in a crossing, taking that number of events from the MC simulation sample of the type, and summing the charge in each channel of the luminosity monitor. A threshold cut is then made on each channel, and the North and South multiplicity for one bunch crossing is determined. For a full MC simulation overlaid sample 10,000 bunch crossings are calculated.

The mean $(\mu)$ number of interactions per bunch crossing for a particular type (XD) of inelastic event is given by Equation 3.6. 


$$
\mu_{X D}=\sigma_{X D} * L /(36 * \nu)
$$

The sigma for each type is given in Equation 3.5. The luminosity, L, is divided by the number of bunches, 36, multiplied by the crossing frequency of the beam, $\nu=47.7 \mathrm{kHz}$. Since the number of events can fluctuate about this mean, Poisson statistics are used to determine how many events of each type are used in a given bunch crossing. In some cases, the number of events of a particular type needed to make an overlaid sample was more than the number of events created in the type specific MC simulation sample set. Care was taken not to bias the overlaid sample by insuring that events from each of the type specific MC sample sets was not used more than twice.

\subsection{Data Samples}

One important difference between the preliminary Run IIB luminosity constant determination and the final determination was the data samples used. The first time the Run IIB constant was determined the data samples, referred to as NTuples, were taken from the online monitoring program lmExamine. Six NTuples, see Table 3.2, over a range of luminosities from 15E30 to 150E30 were used. In the full Run IIB determination of the luminosity constant, high statistics data taken directly from the luminosity system electronics was used. As discussed in Section 2.3.2, a network connection allows direct communication with the VTX board in the VME crate. High 
statistics 2D histograms of the in-time hits for the North versus South arrays were taken for specific ticks, an example of which is shown in Figure 3.7. Recall from Section 1.2 that the 36 ticks with beam are called bunches and that the bunches are separated by at least 2 empty ticks. The procedure for the high statistics data collection was to collect data for 2 minutes on a background tick, 4 minutes on a data tick, and another 2 minute on the background tick. The data tick used for the luminosity constant determination was tick 7 , which is the first tick with beam after an abort gap, and the corresponding background tick was tick 6. A cross-check of the luminosity constant and further study of the backgrounds were done with data from tick 40, the last tick with beam in the first super-bunch, and the corresponding background was from tick 43 . For the data samples, the counts for the background tick were combined and subtracted from the beam crossing tick. This is necessary since the raw data from the VTX board does not include a background correction. The background is believed to be particles from material activation and slow moving neutrons [21].

\begin{tabular}{|c|c|c|}
\hline Run & Date & Nominal L \\
\hline 237470 & 1 Nov 2007 & $15.2 \mathrm{E} 30$ \\
234317 & 13 July 2007 & $28.5 \mathrm{E} 30$ \\
238227 & 2 Dec 2007 & $47.3 \mathrm{E} 30$ \\
234833 & 1 Aug 2007 & $76.3 \mathrm{E} 30$ \\
230714 & 20 Feb 2007 & $103.6 \mathrm{E} 30$ \\
234333 & 13 July 2007 & $152.8 \mathrm{E} 30$ \\
\hline
\end{tabular}

Table 3.2 : Data samples for the preliminary Run IIB luminosity constant determination [13]. 


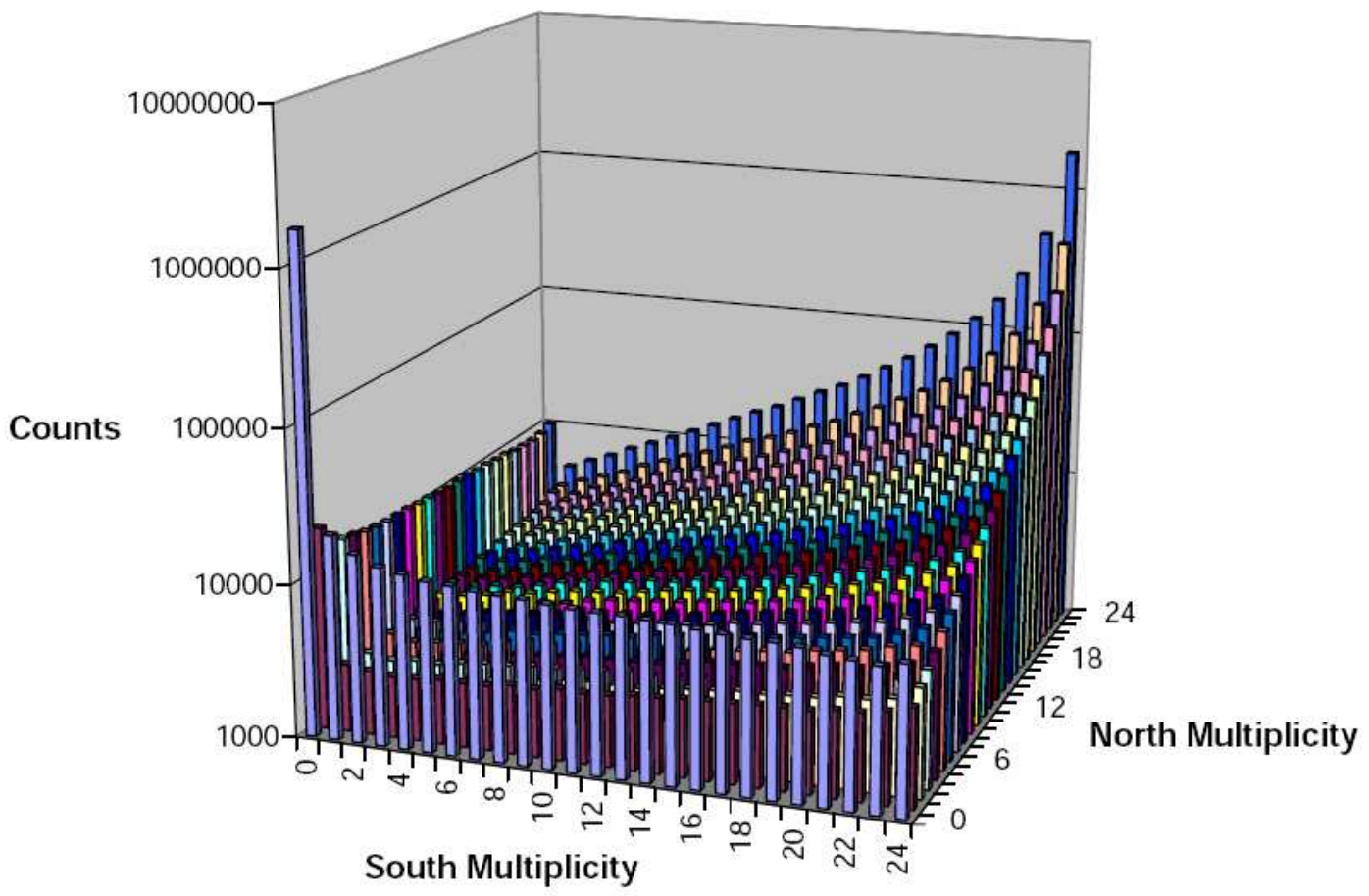

Figure 3.7 : High Statistics 2D histogram collected from the VTX board in the luminosity system at an instantaneous luminosity of 63 E30. Note the peak at $0-0$, which is due to "empty crossings", the hits with North (South) Multiplicity $=0$ and South (North) hits $>1$, which are single sided events, and the peak of non-diffractive hits at 24-24 hits [21]. 
The 2D histograms are different from the NTuples in a few important ways. The NTuple files from lmExamine combine data for an entire run. Since the data is processed through the $\mathrm{D} \varnothing \mathrm{DAQ}$ system, the information is collected for only a small fraction of beam crossings. This means that the data spans a wider luminosity range, though runs without a large range of luminosities were selected. The NTuple files had significantly lower statistics than the 2D histograms taken from the VTX board. Another difference is that the NTuple files from lmExamine could had from all bunch crossing while the 2D histograms were aquired for specific ticks. The $2 \mathrm{D}$ histograms data sets have high statistics, a small luminosity range for the data, and allow precise modeling of the backgrounds [21].

\subsection{Determining the Non-Diffractive Fraction}

The Run IIA and Run IIB determination of the luminosity constant used similar techniques. In both cases the non-diffractive fraction $\left(f_{N D}\right)$ was determined by comparing data and MC simulations. Multiplicity histograms from data and MC simulations were compared and the non-diffractive fraction was adjusted so that the zero-bin agreed. Multiplicity histograms are constructed requiring at least one hit on the opposite side of the luminosity monitor. The zero-bin is fit to seperate diffractive events, which are most likely to only fire one side of the luminosity monitor, from nondiffractive events, which are highly likely to fire both sides of the luminosity monitor. Figure 3.8 compares multiplicity distributions for Run IIA and Run IIB at similar 
luminosities. The difference in the shape is due only to the amount of material added between the beam interaction region and the luminosity monitors.

The first determination of the Run IIB luminosity constant used NTuple data samples from lmExamine and MC simulation samples that only included the Layer 0 sensor material and upgrades to the beam pipe and beam pipe support structure. To improve statistics North and South distributions were added together in both data and MC simulations. The MC simulation samples of each type, from which the overlays that duplicate beam conditions were created, only had 6000 interactions. For the preliminary constant, the data and MC simulation multiplicity distributions were overlaid and $f_{N D}$ was varied over a reasonable range. For each value of $f_{N D}$, the $\chi^{2}$ between the data and the MC simulation was calculated and the value of $f_{N D}$ that gave the minimum $\chi^{2}$ was taken. An example of the $\chi^{2}$ fit values is shown in Figure 3.9. This process was repeated for data taken at several different luminosities to ensure that the $f_{N D}$ was constant for a wide range of luminosity values. The result, displayed in Figure 3.10, was a non-diffractive fraction equal to $0.679 \pm 0.008$ [13].

For the full determination of the Run IIB luminosity constant, the data was taken from the 2D histograms of tick 7 , the MC simulation included all the geometry upgrades shown in Section 3.3.1, and the samples for each type had 10,000 events. The comparison between data and MC simulation was done using a 2D fit that simultaneously determined both the luminosity $(\mathrm{L})$ and the non-diffractive fraction $\left(f_{N D}\right)$. In this study, the values of $f_{N D}$ were determined for both the North and South arrays of 


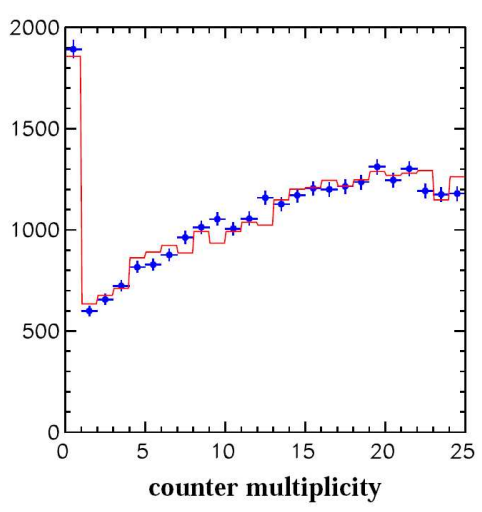

(a) Run IIA

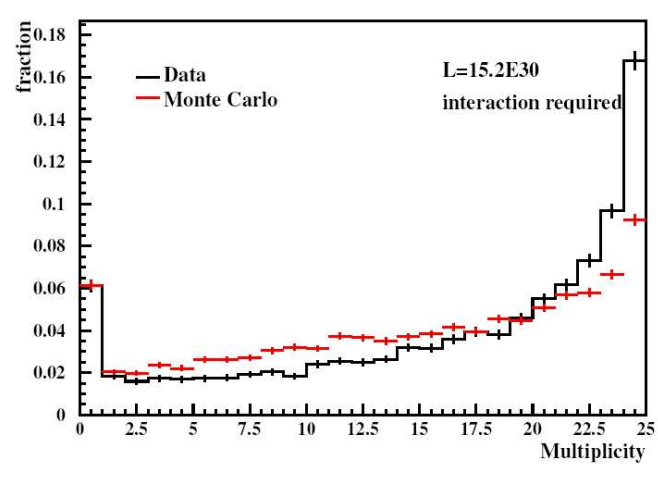

(b) Run IIB

Figure 3.8 : Plots showing the difference in the Run IIA and Run IIB multiplicity distributions due to the addition of material associated with Layer 0. The Run IIA distribution was taken at a luminosity of 13E30, and the Run IIB plot is for a luminosity of 15E30 [7], [13].

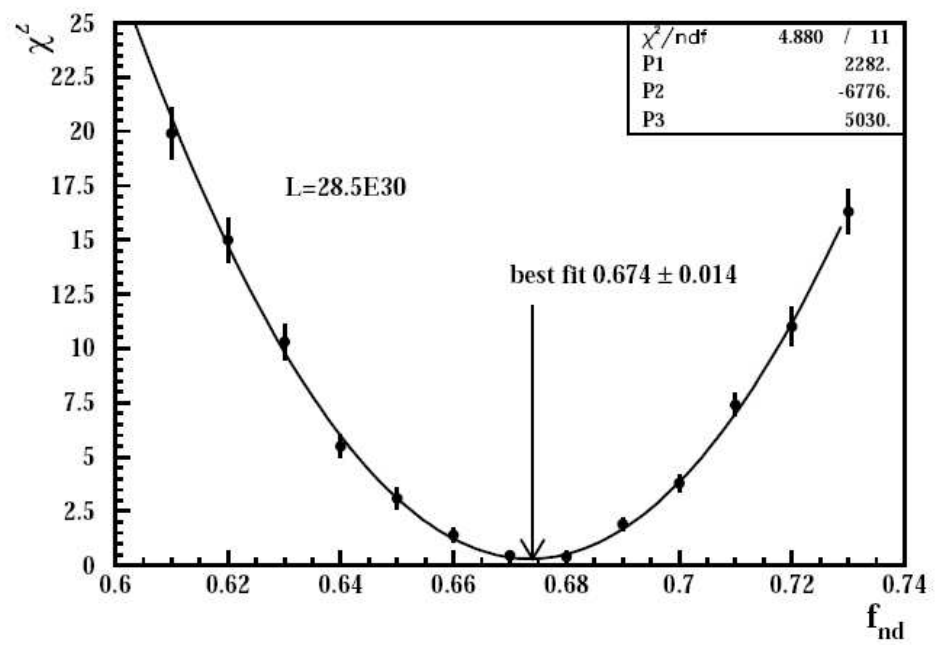

Figure 3.9 : Plot of the $\chi^{2}$ for a range of $f_{N D}$ values at a luminosity of $28.5 \mathrm{E} 30$ for the preliminary Run IIB luminosity constant determination. Each point required a making new MC simulation overlaid sample to be compared with the data [13]. 


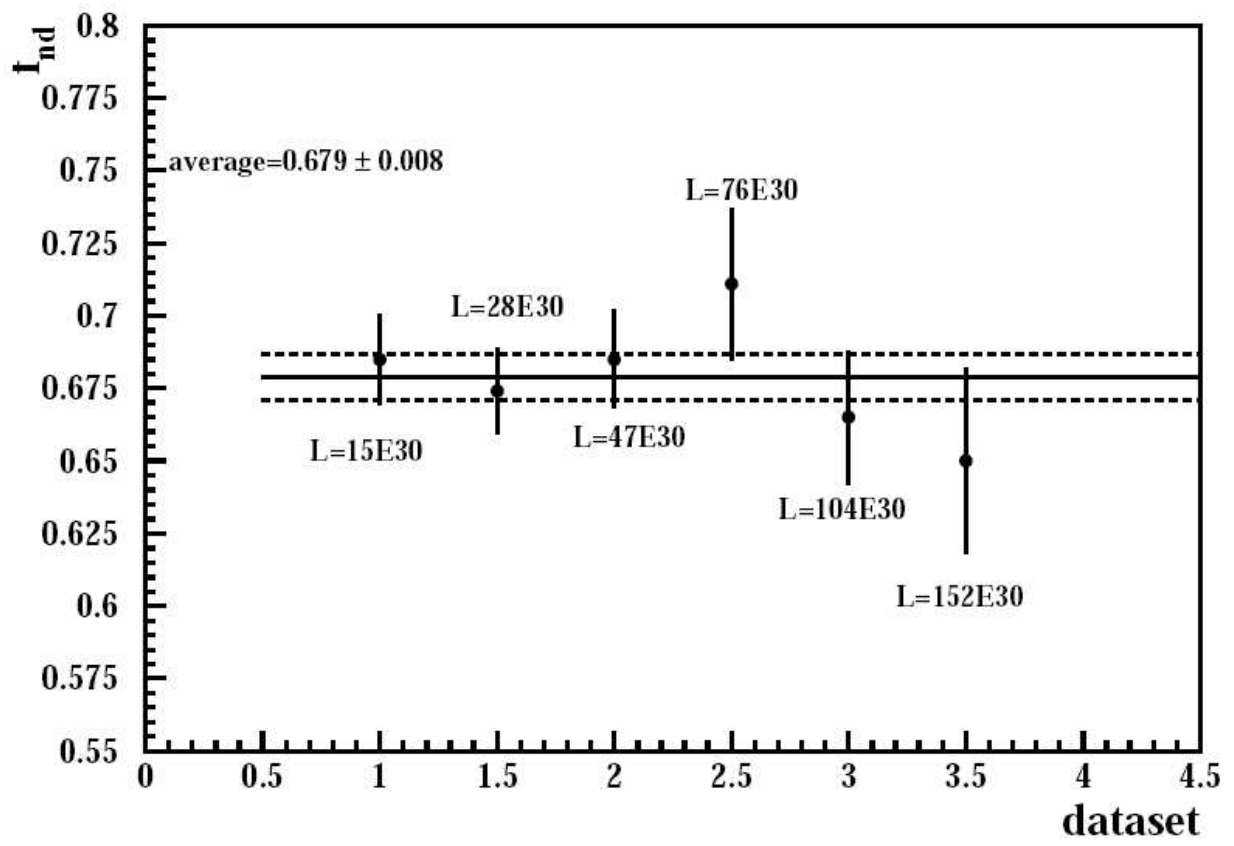

Figure 3.10 : Plot of the values of the non-diffractive fraction $\left(f_{N D}\right)$ for the different luminosity data samples used in the preliminary Run IIB luminosity constant determination [13]. 
the luminosity monitor, as shown in Figure 3.11, which was only possible because the 2D histograms had approximately 1000 times better statistics than the data samples used in previous analyses. The non-diffractive fraction was taken as an average of the North and South values and found to be $0.668 \pm 0.002$ (RMS). The asymmetry in North and South is believed to be due to halo contributions and was taken as part of the systematic uncertainty [21]. The full determination of the luminosity constant also included a detailed study of the background contribution to the luminosity measurement, which can be found in DØNote 5946 [12].

\subsection{Results for $\sigma_{e f f}$}

The preliminary determination of the Run IIB luminosity constant determined that $\sigma_{e f f}=48.3 \pm 0.5 \mathrm{mb}$ and $\sigma_{S S}=9.14 \pm 0.11 \mathrm{mb}$ where the error on both numbers is purely statistical. The study found that the values were consistent within statistical errors to the Run IIA values so that the Run IIA values continued to be used along with the $6.1 \%$ uncertainty [13].

The full Run IIB luminosity constant determination included calculating $\sigma_{\text {eff }}$ and $\sigma_{S S}$ as well as a study of the backgrounds and the systematic uncertainty. The Run IIB luminosity constant is given in Equation 3.7 and the single-sided cross section is given in Equation 3.8 [21].

$$
\sigma_{e f f}=\sigma_{I N} *\left(f_{N D} * A_{N D}+f_{S D} * A_{S D}+f_{D D} * A_{D D}\right)=48.3 \mathrm{mb}
$$




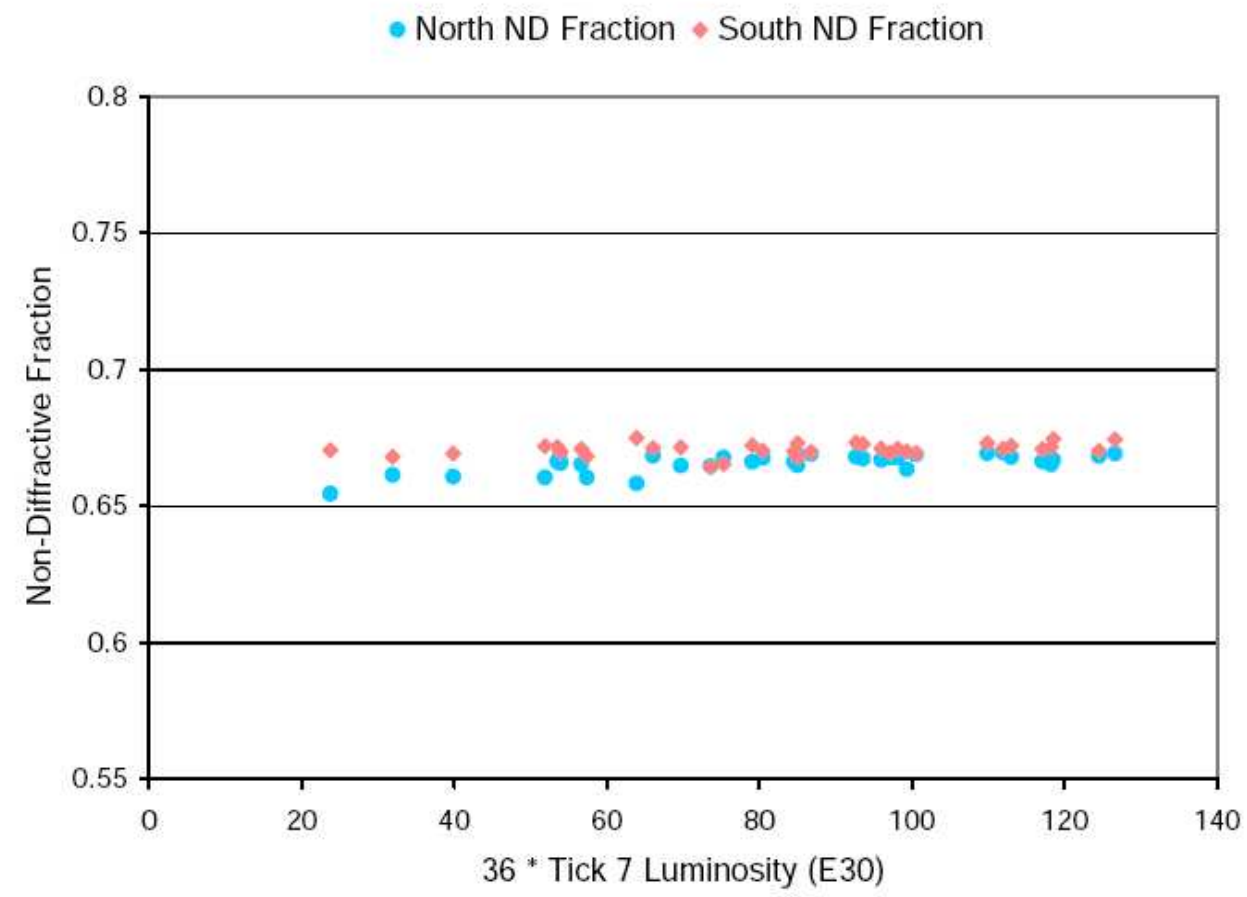

Figure 3.11 : Plot of the non-diffractive fraction $\left(f_{N D}\right)$ values as a function of luminosity for the full Run IIB luminosity constant determination. The values of $f_{N D}$ were determined for both the North and South luminosity monitor arrays, and then the average value was taken $[21]$. 


$$
\sigma_{S S}=\sigma_{N O}+\sigma_{S O}=4.5 \mathrm{mb}+4.5 \mathrm{mb}=9.0 \mathrm{mb}
$$

\subsection{Systematic Studies}

The preliminary Run IIB luminosity constant analysis did not include a study of the systematics. A second material model was used to get better agreement between the data and the MC simulation over the full multiplicity distribution, which showed that the effect to the luminosity constant was minimal. A similar procedure had been used in the Run IIA luminosity constant analysis to show that the fit of the zero-bin is most important [13].

The goal of the full analysis was to more precisely measure the constant for Run IIB and to study the systematic uncertainties, possibly reducing these values. In order to determine the uncertainty of the luminosity constant, the necessary changes were made and propagated through the entire analysis chain including generating new MC samples, re-determining the acceptances and non-diffractive fraction, and finding the value of the constant. The largest uncertainties in the luminosity constant are from the inelastic cross section and the single diffractive/double diffractive ratio. The error from these sources cannot be reduced. The other sources of uncertainty are divided into two categories, contributions from the data and contributions from the MC simulation [21]. 


\subsubsection{Uncertainty Associated with Data}

The largest uncertainty associated with the data used in the full determination of the Run IIB luminosity constant is the variation in the measured luminosity over time. As discussed in Section 2.3.3, the HV on the PMTs is periodically increased to keep the gains stable and keep the luminosity measurement within a $0.5 \%$ stability range. This translates into a $0.24 \mathrm{mb}$ uncertainty in the luminosity constant [21].

When calculating $f_{N D}$ from the high statistics histograms, a North/South asymmetry and luminosity dependence was seen in the data, which is shown in Figure 3.11. The North/South asymmetry could be explained by a slight offset in the distance from the beam interaction point to the luminosity monitors. The End Cap (EC) Calorimeters on which the luminosity monitor arrays sit are not symmetrically spaced, and the difference is on the order of $2 \mathrm{~cm}$. The differences seen in $f_{N D}$ are taken as a systematic uncertainty [21].

Data for tick 7 was used to calculate $f_{N D}$ while data from tick 40 was used as a cross check. Because tick 40 is at the end of a super bunch, it has larger backgrounds from a build up of slow neutrons and activation particles from the previous bunch crossings. The difference between the values of $f_{N D}$ for tick 7 and tick 40 were propagated to the luminosity constant and taken to be an uncertainty due to the background unfolding technique [21].

The other data-related uncertainties can be grouped together as operational variations that occur in the data but were tested using the MC simulation. This group 
includes differences seen due to the seasonal timing shift, explained in Section 2.3.1, variation of the charge threshold about the nominal, and the effect of the radiation damage to the scintillator on the luminosity measurement. The timing was shifted by \pm 1 ns in the MC simulation to reflect the seasonal fluctuations before a calibration change is made. The charge threshold was varied by $\pm 2 \mathrm{pc}$ about the nominal value of $8 \mathrm{pC}$. To simulate the radiation damage to the scintillating wedges, the light collection efficiency was reduced by a factor of 2 at the inner and outer edge of the scintillator and scaled linearly from each edge so that no reduction in light was seen at the at the PMT [21].

\subsubsection{Uncertainty Associated with Monte Carlo Simulation}

For the full Run IIB luminosity constant determination, 10,000 interactions of each type of inelastic event, $\mathrm{ND}, \mathrm{SD}$, and $\mathrm{DD}$, were produced for every variation to the $\mathrm{MC}$ simulation. The statistical uncertainty associated with this finite sample size, $0.06 \mathrm{mb}$, is one source of $\mathrm{MC}$ simulation uncertainty. The other factors that contribute to the luminosity constant uncertainty from the MC simulation come from differences seen when variations are made to parameters at the geometry and generator level [21].

While it is most important to fit the zero-bin of the multiplicity distributions between data and MC simulations, changes to the MC simulation were made to get a better fit over the entire multiplicity range, and the differences were taken to be systematic uncertainties. After all the known material in the forward region was added 
to the geometry, the MC simulation still underestimated the high multiplicity region. It is possible that some material is missing from areas like the silicon detector. Figure 3.12 shows the multiplicity distribution comparing data and the nominal material model. To get full multiplicity agreement, the beryllium beam pipe was changed to tin, see Figure 3.13, which is an addition of approximately 0.7 radiation lengths at a pseudo-rapidity of $\eta=4$. This technique was also used in the preliminary Run IIB and the Run IIA determination [21].

Another adjustment done in an attempt to get full multiplicity agreement was tuning Pythia parameter PARP(82). This parameter adjusts the transverse momentum $\left(p_{T}\right)$ value at which the parton-level cross section $\left(\frac{d \sigma}{d t}\right)$ starts to decrease toward zero. In reality, the cross section blows up $\left(\frac{d \sigma}{d t} \rightarrow \infty\right)$ as the momentum transfer goes to zero $(t \rightarrow 0)$. The turn down for low $p_{T}$ is put into Pythia "by hand". The value was adjusted from the default of $2.0 \mathrm{GeV}$, which is used for physics analysis, down to $1.25 \mathrm{GeV}$, shown in Figure 3.14, which gives agreement at high multiplicity [21].

The following changes to the MC model were made to determine if the fit was dependent on specific parameters used to generate the MC simulation samples. The Parton Distribution Function (PDF) was changed to MRST2004NLO, which includes a next to leading order correction, from the default of CTEQ6L1. The GEANT hadronic model GEISHA was tested as well as the default of GCalor. Another change to the GEANT simulation was lowering the energy cut offs. GEANT tracks most particles down to an energy of $1 \mathrm{MeV}$ and then drops the particle from the simulation. 


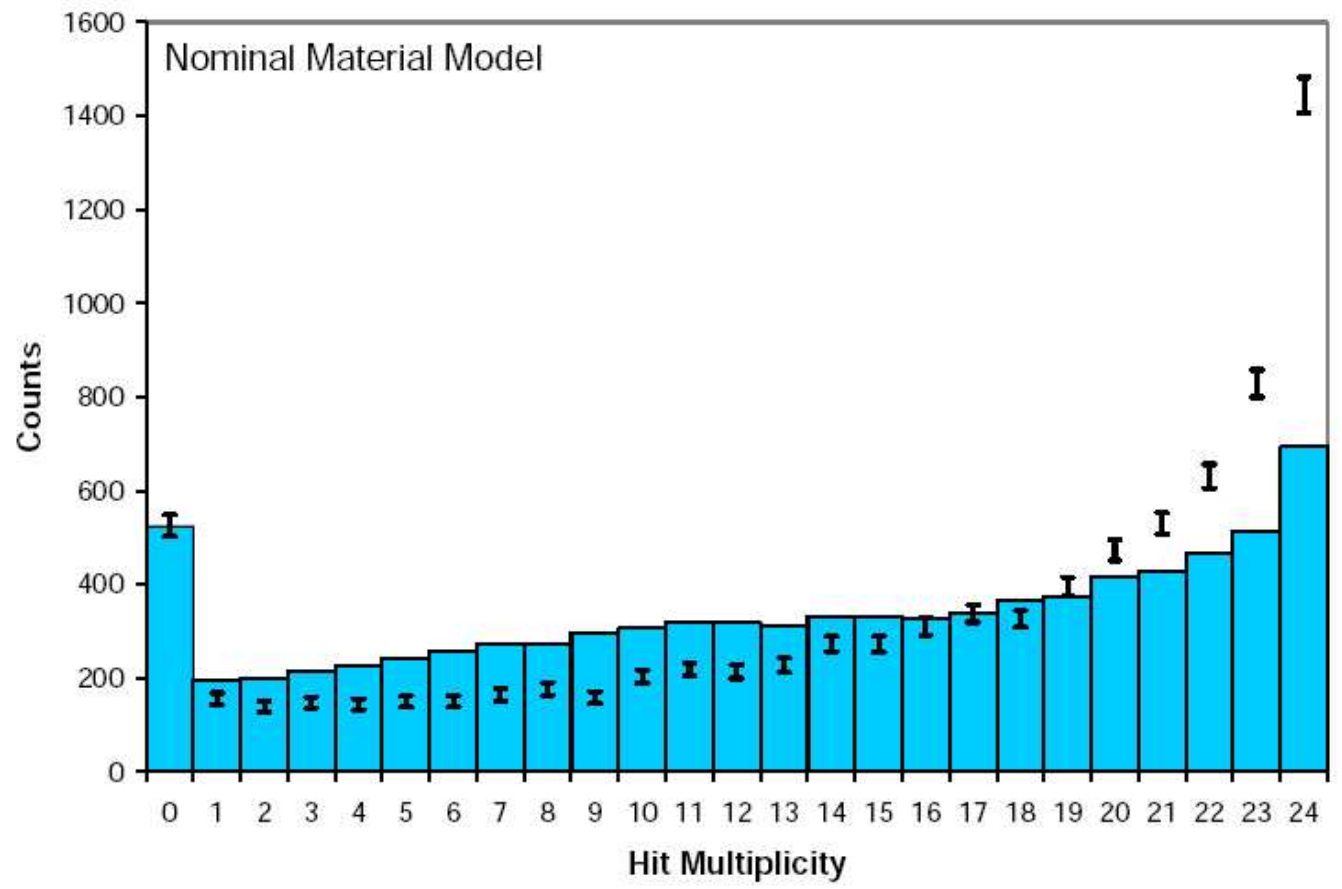

Figure 3.12 : Multiplicity distribution comparing data (black) and the nominal material model MC simulation (blue) at a luminosity of 15E30 [21]. 


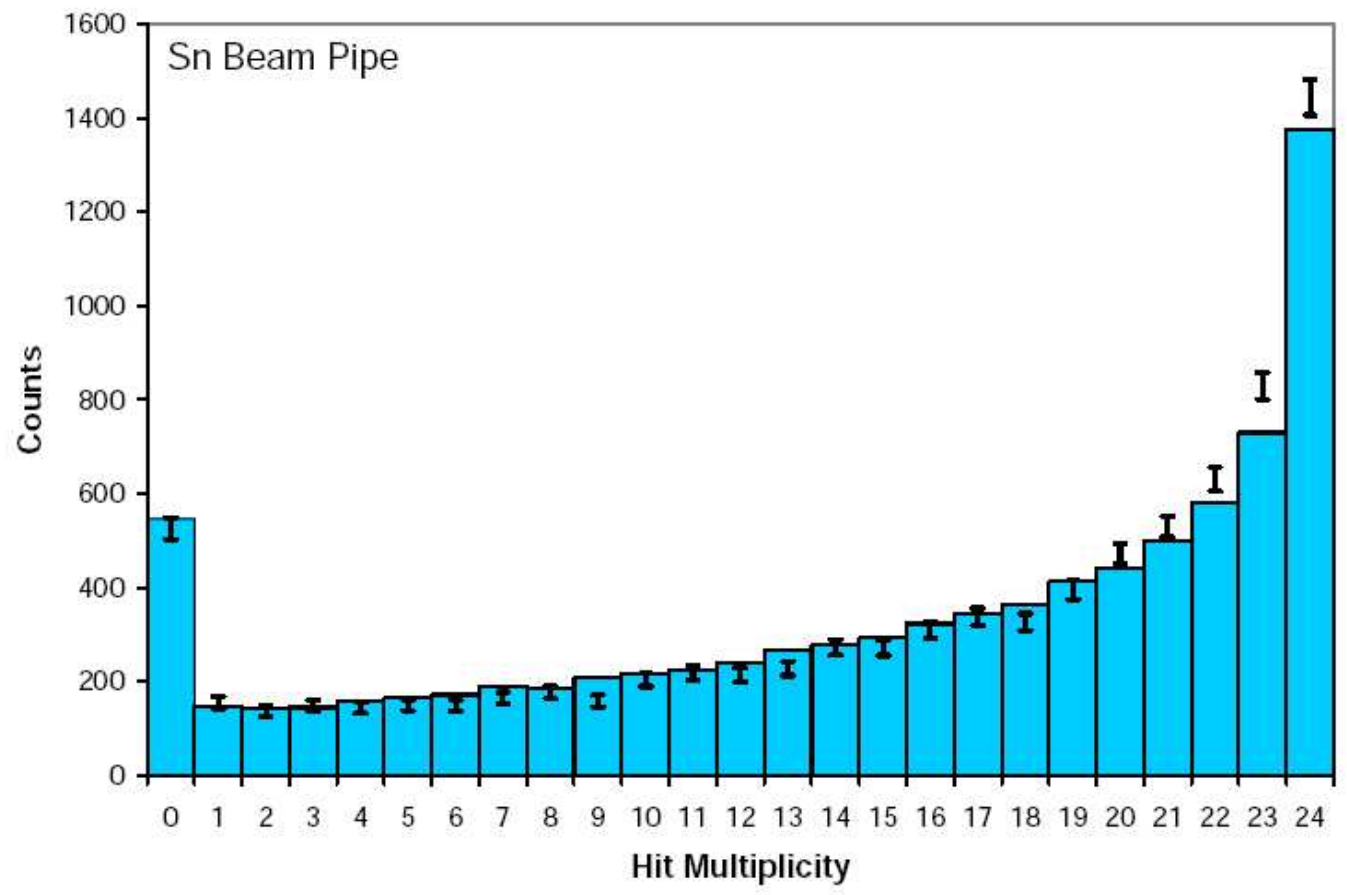

Figure 3.13 : Multiplicity distribution comparing data (black) and a geometry with a tin beam pipe in the MC simulation (blue) to get better full multiplicity agreement at a luminosity of 15E30 [21]. 


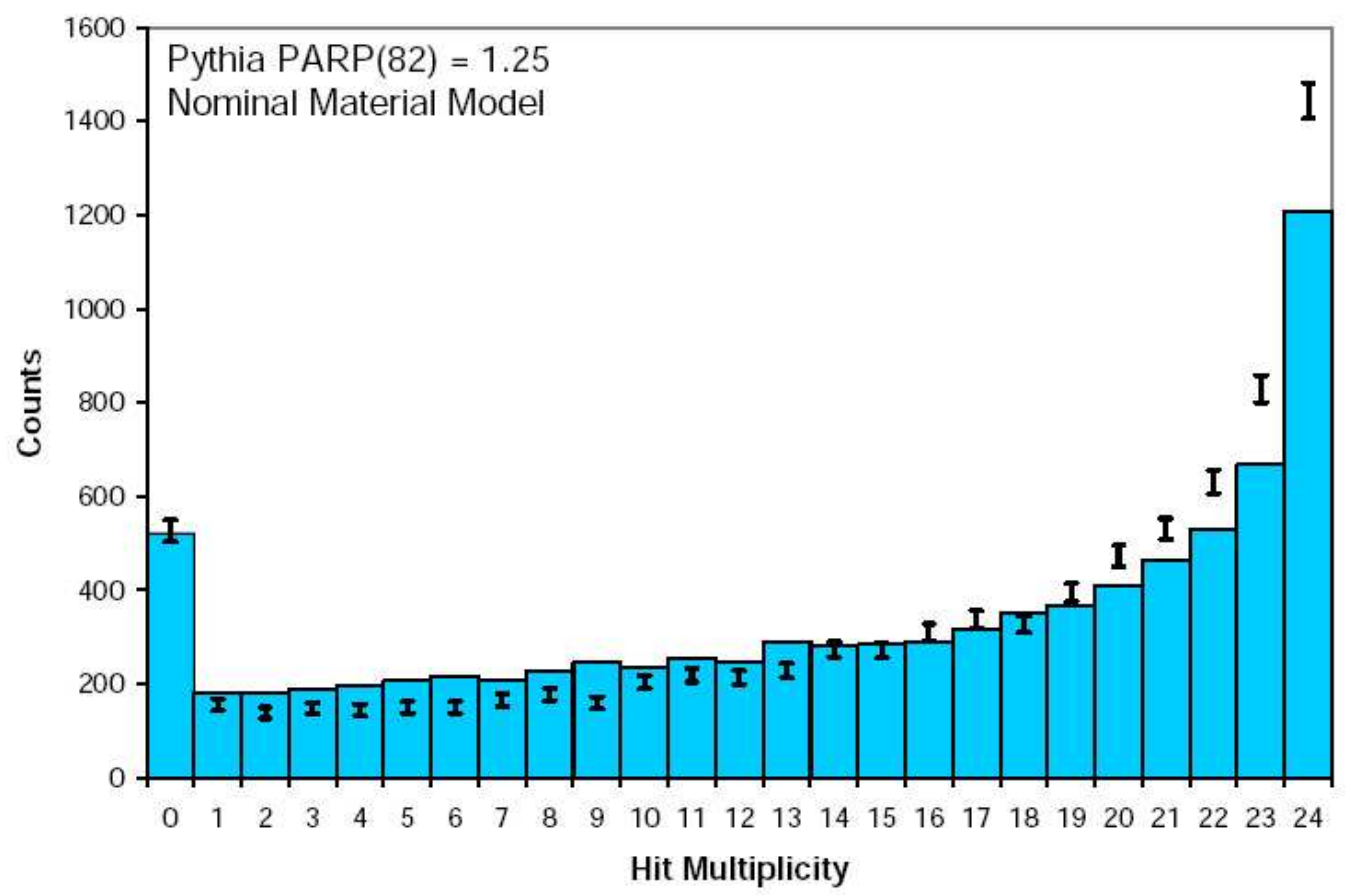

Figure 3.14 : Multiplicity distribution comparing data (black) and a MC simulation (blue) with the transverse momentum $\left(p_{T}\right)$ of the parton-level cross section adjusted from $2 \mathrm{GeV}$ to $1.25 \mathrm{GeV}$ to get better full multiplicity agreement at a luminosity of $15 \mathrm{E} 30$ [21]. 
Since the luminosity monitor can have multiple hits in one channel that add together to pass the threshold cut but which would not individually fire as a hit, it was important to make sure the energy cuts in GEANT are low enough to allow this possibility. The value of $f_{N D}$ for a MC simulation sample set in which GEANT tracked particles down to energies as low as $10 \mathrm{keV}$ was used to determine the luminosity constant, and the difference was taken as a systematic uncertainty [21].

Table 3.3 shows the values of all the contributions to the uncertainty of the luminosity constant listed in order of magnitude [21].

\subsection{Conclusions}

The Run IIB luminosity constant is given in Equation 3.9.

$$
\sigma_{\text {eff }}=48.3 \mathrm{mb} \pm 1.9 \mathrm{mb} \pm 0.6 \mathrm{mb}
$$

The uncertainty of $1.9 \mathrm{mb}$ is associated with the inelastic cross section that has been agreed upon by CDF and DØ. This value was the same in the Run IIA analysis of the luminosity constant and cannot be reduced. The uncertainty of $0.6 \mathrm{mb}$ is specific to $\mathrm{D} \varnothing$. This work represents a reduction in the $\mathrm{D} \varnothing$ specific uncertainty from $4.6 \%$ to $1.3 \%[21]$

The $\mathrm{D} \varnothing$ luminosity system is a robust detector that is crucial to physics at D $\varnothing$.

With careful maintenance the LM will provide an accurate luminosity measurement within the reduced uncertainty associated with the luminosity constant. 


\begin{tabular}{|l|c|}
\hline Source & Uncertainty \\
\hline Inelastic Cross-Section & $\pm 1.91 \mathrm{mb}$ \\
Single Diffractive Fraction & $\pm 0.43 \mathrm{mb}$ \\
Time Variation / Radiation Damage & $\pm 0.24 \mathrm{mb}$ \\
GEANT Energy Cutoffs & $\pm 0.24 \mathrm{mb}$ \\
Monte Carlo Material Model & $\pm 0.16 \mathrm{mb}$ \\
Light Collection / Radiation Damage & $\pm 0.09 \mathrm{mb}$ \\
North / South Asymetry & $\pm 0.09 \mathrm{mb}$ \\
Luminosity Dependence & $\pm 0.08 \mathrm{mb}$ \\
Monte Carlo Statistics & $\pm 0.06 \mathrm{mb}$ \\
PDF Choice & $\pm 0.06 \mathrm{mb}$ \\
Pythia Tune & $\pm 0.04 \mathrm{mb}$ \\
Background Unfolding & $\pm 0.03 \mathrm{mb}$ \\
GEANT Hadronic Model & $\pm 0.03 \mathrm{mb}$ \\
Seasonal Timing Variation & $\pm 0.02 \mathrm{mb}$ \\
Charge Threshold & $\pm 0.01 \mathrm{mb}$ \\
\hline
\end{tabular}

Table 3.3 : List of uncertainties for the Run IIB luminosity constant. The largest uncertanties from the inelastic cross-section and single diffractive fraction cannot be reduced. When added in quadrature the total uncertainty is $2.0 \mathrm{mb}$ [21]. 


\section{Bibliography}

[1] F. Halzen and A. D. Martin. Quarks \& Leptons: An Introductory Course in Modern Particle Physics. John Wiley \& Sons, New York, 1984.

[2] S. Abachi et al. Observation of the Top Quark. Phys. Rev. Letters, 74(14).

[3] Fermilab Accelerator Division. Concepts Rookie Book, 2010. http://wwwbdnew.fnal.gov/operations/rookie_books/rbooks.html.

[4] Fermilab Accelerator Division. Antiproton Source Rookie Book, 2009. http://www-bdnew.fnal.gov/operations/rookie_books/rbooks.html.

[5] Fermilab Accelerator Division. Tevatron Rookie Book, 2009. http://wwwbdnew.fnal.gov/operations/rookie_books/rbooks.html.

[6] V.M. Abazov et al. The Upgraded DØ Detector. Nucl. Instr. and Methods, A565:463, 2006.

[7] B. Casey, Y. Enari, T. Kuhl, T. Nunnemann, R. Partridge, P. Polozov, H. Schellman, and M. Wobisch. Determination of the Effective Inelastic $p \bar{p}$ Cross-Section for the DØ Luminosity Measurement Using Upgraded Readout Electronics. Internal Note 4958, DØ Collaboration, 2005.

[8] Saint-Gobain Crystals. Premium Plastic Scintillators, 2005. http://www.detectors.saint-gobain.com/Plastic-Scintillator.aspx.

[9] Hamamatsu. Fine Mesh PMT Series for High Magnetic Field Environments, 2000. ams.bo.infn.it/PMT_Fine_Mesh.pdf.

[10] J. Anderson, L. Bridges, B. Casey, Y. Enari, J. Green, M. Johnson, R. Kwarciany, C. Miao, R. Partridge, H. D. Yoo, and J. Wang. Upgrade of the D $\varnothing$ Luminosity Monitor Readout System. Internal Note 5297, DØ Collaboration, 2006.

[11] R. Brun, O. Couet, C. Vandoni, and P. Zanarini. PAW - Physics Analysis Workstation. CERN Program Library Long Writeup Q121, 2009.

[12] Y. Enari, R. Partridge, and M. Prewitt. Luminosity Monitor Background Cross Section. Internal Note 5946, DØ Collaboration, 2009. 
[13] B. Casey, M. Corcoran, Y. Enari, M. Prewitt, and G. Snow. Determination of the DØ Run IIB Luminosity Constants. Internal Note 5559, DØ Collaboration, 2007.

[14] F. Abe et al. Measurement of the Antiproton-Proton Total Cross Section at $\sqrt{s}=546$ and $1800 \mathrm{GeV}$. Phys. Lett., D50:5550, 1994.

[15] N. A. Amos et al. Measurement of the $p \bar{p}$ Total Cross Section at $\sqrt{s}=1.8 \mathrm{TeV}$. Phys. Rev. Letters, 63:2784, 1989.

[16] C. Avila et al. A Measurement of the Proton-Antiproton Total Cross Section at $\sqrt{s}=1.8 \mathrm{TeV}$. Phys. Lett., B445:419, 1999.

[17] H.-U. Bengtsson and T. Sjöstrand. The Lund Monte Carlo for hadronic processes - PYTHIA version 4.8. Comp. Phys. Comm., 46:43, 1987.

[18] R. Field. Pythia Tune Set A, 2002. http://www.phys.ufl.edu/〜rfield/cdf/tunes/ py_tuneA.html.

[19] R. Brun et al. GEANT 3 - Detector Description and Simulation Tool. CERN Program Library Long Writeup W5013, 1993.

[20] B. Casey, Y. Enari, K. DeVaughan, R. Partridge, and S. Yacoob. Radiation Damage to Scintillator in the DØ Luminosity Monitor. Internal Note 5255, DØ Collaboration, 2006.

[21] B. Casey, M. Corcoran, Y. Enari, R. Partridge, M. Prewitt, and G. Snow. The DØ Run IIB Luminosity Constant. Internal Note 5945, DØ Collaboration, 2009. 